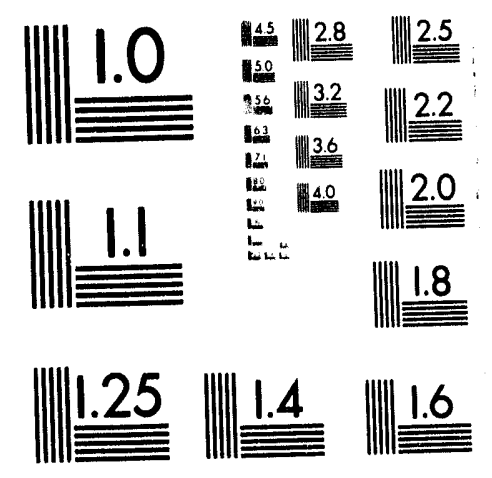



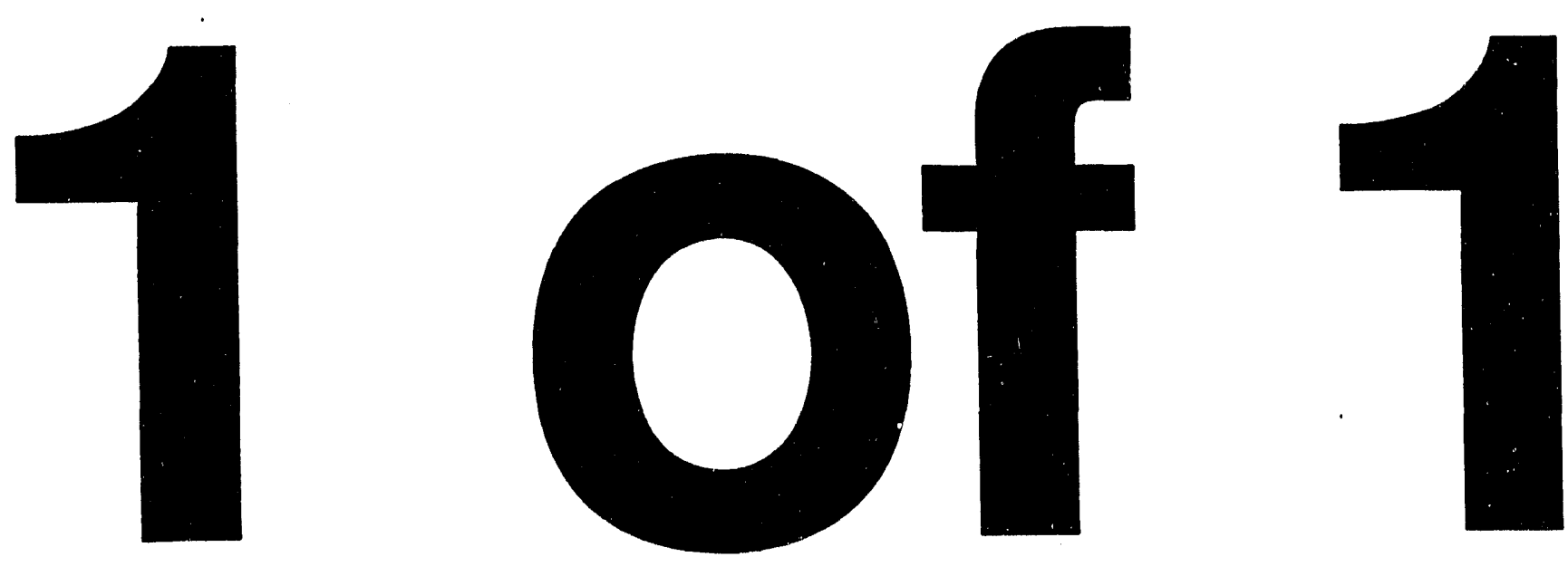
Engineering Physics and Mathematics Division Mathematical Sciences Section

\title{
A METHOD FOR ESTIMATING
} OCCUPATIONAL RADIATION DOSE

TO INDIVIDUALS, USING WEEKLY DCSIMETRY DATA

\author{
Toby J. Mitchell a \\ George Ostrouchov * \\ Edward L. Frome ${ }^{a}$ \\ George D. Kerr ${ }^{\circ}$ \\ - Mathematical Sciences Section \\ Engineering Physics and Mathematics Division \\ Oak Ridge National Laboratory \\ P. O. Box 2008 \\ Oak Ridge, Tennessee 37831-6367 \\ - Assessment Technology Section \\ Health Sciences Research Division \\ Oak Ridge National Laboratory \\ P. O. Box 2008 \\ Oak Ridge, Tennessee 37831-6383 \\ $\dagger$ deceased
}

Date Published: December 1993

Research was supported by the Office of Epidemiology and Health Surveillance, Environment, Safety and Health, U. S. Department of Energy

Prepared by the

Oak Ridge National Laboratory

Oak Ridge, Tennessee 37831

operated by

Martin Marietta Energy Systems, Inc.

for the

U.S. DEPARTMENT OF ENERGY

under Contract No. DE-AC-05-840R21400 


\section{Contents}

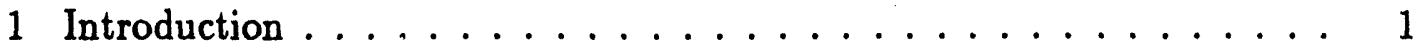

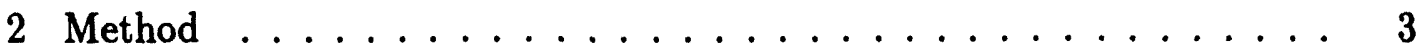

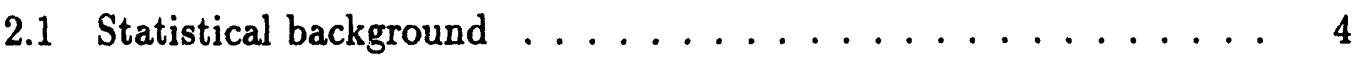

2.2 Example ....................... 6

3 Estimating Yearly Dose . . . . . . . . . . . . . . . 14

4 Use of Other Data . . . . . . . . . . . . . . . . . 18

4.1 Modifying the Likelihood . . . . . . . . . . . . . . . . 19

4.2 Specifying the Prior . . . . . . . . . . . . . . 22

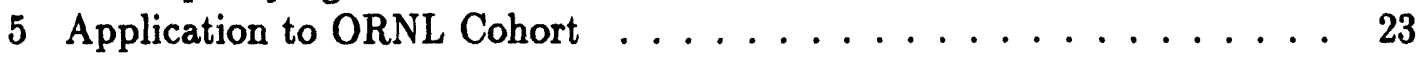

5.1 Dose Estimation . . . . . . . . . . . . . . . 25

5.2 Dose-Response Analysis . . . . . . . . . . . . . . . 29

5.3 Computational Issues . . . . . . . . . . . . . . . . 30

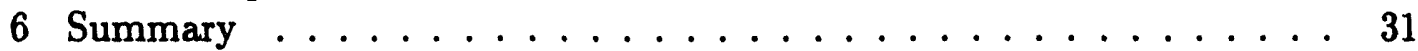

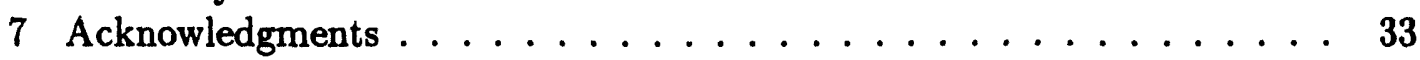

8 References . . . . . . . . . . . . . . . . . 33 


\section{List of Figures}

1 Probability density function for prior distribution of $x$. . . . 7

2 Distribution function for prior distribution of $x \ldots \ldots 8$

$3 \quad \alpha$, the standard deviation of $\log (\tilde{z})$ versus true dose. . . . . . . . 10

$499 \%$ probability bounds on expressed dose $(\tilde{z})$ versus true dose $(x) .11$

5 Probability density function of expressed dose $\tilde{z}$ when true dose $x$ is $0.32 \mathrm{mSv} \ldots \ldots \ldots \ldots \ldots \ldots \ldots$

$6 \quad$ Probability density of recorded dose $z$ when true dose $x$ is $0.32 \mathrm{mSv} .12$

$7 \quad$ Likelihood of true dose $x$ wh $3 n$ recorded dose $z$ is $0.40 \mathrm{mSv}$. . . 13

8 Posterior distribution of true dose $x$ when recorded dose $z$ is 0.40 mSv. ............................. 13

9 Comparison of prior to posterior distribution of true dose $x$ when recorded dose $z$ is $0.40 \mathrm{mSv}$. . . . . . . . . . . . . . . 14

10 Likelihood of true dose $x$ when recorded dose $z$ is zero. . . . . . 15

11 Posterior distribution of true dose $x$ when recorded dose $z$ is zero. 15

12 Comparison of prior to posterior distribution of true dose $x$ when recorded dose $z$ is zero. . . . . . . . . . . . . 16

13 Yearly dose distribution estimate for person A (with recorded dose of $0.65 \mathrm{mSv}$ ) based on a fixed prior that assumes he is a radiation worker with a true weekly dose of about one tenth of the occupational limit. . . . . . . . . . . . . . . . . .

14 Yearly dose distribution estimates for person B (with recorded dose of $27.2 \mathrm{mSv}$ ) based on a fixed prior that assumes he is a radiation worker with a true weekly dose of about one tenth of the occupational limit. . . . . . . . . . . . . . .

15 Likelihood of true dose $x$ when recorded dose $z$ is zero without

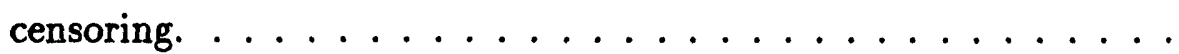

16 Comparison of prior and posterior distributions of true dose $x$ when recorded dose $z$ is zero without censoring. . . . . . . . . . .

17 Yearly dose distribution estimate for person $A$ (with recorded dose of $0.65 \mathrm{mSv}$ ) allowing "rounded" zeros and with a fixed prior. . . Yearly dose distribution estimate for person $B$ (with recorded dose of $27.2 \mathrm{mSv}$ ) allowing "rounded" zeros and with a fixed prior. . .

19 Yearly dose distribution estimate for an individual with zero recorded film-badge dose and all zero weekly pocket-meter doses. .

20 Yearly dose distribution estimate for person A (with recorded dose of $0.65 \mathrm{mSv}$ ) allowing "rounded" zeros and with pocket-meter specified priors. 
21 Yearly dose distribution estimate for person B (with recorded dose of $27.2 \mathrm{mSv}$ ) allowing "rounded" zeros and with pocket-meter specified priors. ..................... 24

22 Boxplots of yearly dose distribution estimates and the corresponding recorded dose $(\mathrm{mSv})$ for a sample of the ORNL cohort. . . . . 26

23 Boxplots of yearly dose distribution estimates and the corresponding recorded dose ( $\mathrm{mSv}$ ) for a sample of the ORNL cohort (continued). . . . . . . . . . . . . . . . .

24 Boxplots of yearly dose distribution estimates and the corresponding recorded dose $(\mathrm{mSv})$ for a sample of the ORNL cohort (con-

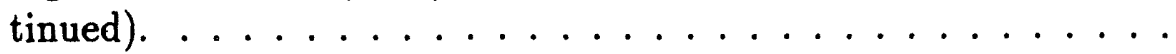

25 Boxplots of yearly dose distribution estimates and the corresponding recorded dose $(\mathrm{mSv})$ for a sample of the ORNL cohort (continued $). \ldots \ldots \ldots \ldots \ldots$ 


\title{
A METHOD FOR ESTIMATING OCCUPATIONAL RADIATION DOSE TO INDIVIDUALS, USING WEEKLY DOSIMETRY DATA
}

\author{
Toby J. Mitchell \\ George Ostrouchov \\ Edward L. Frome \\ George D. Kerr
}

\begin{abstract}
Statistical analyses of data from epidemiologic studies of workers exposed to radiation have been based on recorded annual radiation doses. It is usually assumed that the annual dose values are known exactly, although it is generally recognized that the data contain uncertainty due to measurement error and bias. We propose the use of a probability distribution to describe an individual's dose during a specific period of time. Statistical methods for estimating this dose distribution are developed. The methods take into account the "measurement error" that is produced by the dosimetry system, and the bias that was introduced by policies that lead to right censoring of small doses as zero. The method is applied to a sample of dose histories obtained from hard copy dosimetry records at Oak Ridge National Laboratory (ORNL). The result of this evaluation raises serious questions about the validity of the historical personnel dosimetry data that is currently being used in low-dose studies of nuclear industry workers. In particular, it appears that there was a systematic underestimation of doses for ORNL workers. This could result in biased estimates of dose-response coefficients and their standard errors.
\end{abstract}




\section{Introduction}

In December 1941 at the University of Chicago, the first uranium-graphite pile achieved criticality, and plans were soon underway to construct larger uraniumgraphite piles at Oak Ridge, Tennessee, and at Hanford, Washington $[1,16]$. The purpose of the so-called Clinton Laboratories pilot plant at Oak Ridge was to train crews to operate the even larger production facilities at Hanford and to demonstrate the safe production and chemical separation of the fissionable $\mathrm{Pu} 239$ isotope from uranium irradiated in the so-called X-10 pile or Graphite Reactor at the Clinton Laboratories [10]. The Clinton Laboratories were renamed the Clinton National Laboratory in 1947 and the Oak Ridge National Laboratory (ORNL) in 1948.

Construction was started on the ORNL Graphite Reactor in January 1943 and criticality was achieved in November 1943. The first batch of uranium irradiated slugs from the reactor entered chemical separation at the pilot plant in December 1943. By the end of December, several milligrams of plutonium were separated and shipped for experimentation at the University of Chicago, and by March 1943, gram quantities of plutonium were being made available for experimentation at Los Alamos. After the production facilities became operational at Hanford in September 1944, the ORNL Graphite Reactor was used primarily for fundamental nuclear research and production of medically important radioisotopes.

In the beginning at Chicago and later at Oak Ridge, pocket ionization chambers (or pocket meters) were considered the primary device for monitoring personnel exposures, with a film dosimeter being only a valuable adjunct [9]. With expanding experience at Oak Ridge and with the startup of the production facilities at Hanford in 1944, this practice was reversed, and the film dosimeter provided the official dose of record, while the pocket meter became the day-today means of monitoring personnel exposures in the workplace [21]. At ORNL, however, the daily pocket-meter readings were also maintained as a part of an individual's dose records [9].

An individual's radiation dose of record at ORNL for external penetrating 
radiation, principally gamma rays, is based on pocket meters from 1943 to July 1944, film badges from then to 1975, and thermoluminscent dosimeters since 1975 [22]. The pocket meters were evaluated daily (minimum detectable limit of $0.02 \mathrm{mSv}$ ), and the film badges were evaluated weekly from July 1944 to July 1956, when quarterly monitoring was initiated (minimum detectable limit of $0.30 \mathrm{mSv}$ ). Several reports have already been published about missing dose at ORNL during the weekly evaluations of film badges $[11,12,13]$. However, there is considerable doubt in the current literature concerning the lower detection limit of the film badge dosimeters [23]. The general issue of uncertainty in individual dose estimates in epidemiologic studies of nuclear industry workers also has been discussed in $[2,7,19,20]$.

The lower limit of detection of the most sensitive film used at ORNL was 0.10 to $.30 \mathrm{mSv}$. A lower detection limit of $0.10 \mathrm{mSv}$ was possible if an experienced technician evaluated the exposed films with special care [14]. During film badge exchange, when hundreds to thousands of films were read in large batches by technicians with widely varying experiences, a lower limit of detection of about $0.30 \mathrm{mSv}$ was about as good as could be expected [15]. In practice, a film badge reading of zero means the radiation dose to the worker was less than $0.30 \mathrm{mSv}$ unless a smaller value is given. Thus, the missing dose from weekly evaluations of the film badges occurred primarily among those workers with the lowest radiationdose estimates between 1944 and $1956[11,12,13]$.

The design of the film badge and its use at ORNL changed considerably over the years. In November 1951, for example, the photo film badge was introduced and all ORNL employees were required to wear a film badge on the job [9]. Prior to November 1951 , only those ORNL employees who entered a radiation area were required to wear a film badge. Two or more filters were used in all ORNL film badges to aid in interpreting the radiation dose and in resolving the difficulty due to the fact that the unshielded films were more sensitive to $x$ rays between 50 and $100 \mathrm{keV}$ than to $\mathrm{x}$ or gamma rays above $200 \mathrm{kev}$ [15]. The film badge readings quoted throughout this report are estimates of the equivalent dose from 
external penetrating radiation at a depth of approximately $1 \mathrm{~cm}$ within the total body or a major portion of the total body.

Typically, epidemiological studies of the effects of external penetrating radiation on worker health have relied on recorded annual doses to the individuals in the population as determined by personnel dosimeters. In the statistical analysis, these dose values have been treated as though they are known exactly, although everyone recognizes that there is some uncertainty due to measurement error and bias. It is usually assumed that the measurement errors "average out" and that the bias is small.

In this report, we consider primarily film badge data. In the situation of practical interest, weekly film-badge readings are typically added up to obtain the annual recorded dose, which is then regarded as "known." The lower limit of detection of the film badge, together with the measurement error in the dosimetry system, could lead to substantiai uncertainty in the recorded annual dose.

\section{Method}

Here, we describe a method of accounting for uncertainty and bias in measurements of an individual's occupational radiation dose with film badges. The emphasis here is on the general concept and the manner of implementing it. Detailed models for some of the key probability distributions are still being refined.

The "true" dose here is taken to be the quantity of radiation encountered by the film badge(s) worn by the individual during the period in question. The relevant and difficult issues involved in estimating the dose to the individual (or, even more relevant and more difficult, the estimation of doses to specific organs) will be considered in subsequent reports. However, we expect that our approach to these problems in the future will be based on the same framework as that described in this report.

Here the basic quantities of interest are, for each week, the true dose to the film badge and the recorded dose. Our objective is to provide the methodology for determining the estimated dose (in terms of a probability distribution) of 
an individual during a year, given the recorded weekly exposure histories for that individual in that year. It is usually impossible to determine the quantities that we want to know, in this case true dose, precisely through measurement. There are unobserved values (true doses) and observed values (recorded film badge readings). We assume there is a relationship between the two and we use this relationship to estimate the true dose. The statistical approach that we shall adopt here provides the means to estimate, using the laws and language of probability, the unobserved quantities given the values of the observed ones. For each quantity of interest, this estimate is expressed in the form of a probability distribution. A point estimate (single "best" value, by some criterion) could be obtained from this distribution, but we shall generally avoid this, since we regard the probability distribution itself as the estimate, and think of any reduction as a loss of information. In particular, if the annual doses are to be used as inputs to a model that relates health effects to radiation dose, it is important that the uncertainty in these values be quantified.

\subsection{Statistical background}

In order to obtain estimates of the unobserved quantities, all quantities of interest, observed and unobserved, are endowed with a joint prior probability distribution that represents (approximately) the state of knowledge about them prior to (or external to) observation or measurement. Then the actual values of the observed measurements are put in, as conditioning information, and the laws of probability are used to find the conditional distribution of the unobserved values given the observed ones. See for example [18] for further background on this approach to estimation.

For simplicity, we first consider only a single exposure period, e.g., one week. There are two quantities of interest:

$x$ the unobserved true dose to the film badge, and

$z$ the recorded dose to the film badge. 
It is important to explain why we consider these quantities as random variables, even though they are deterministic in the sense that they each have a fixed value. For example, there can be only one true value for $x$, but, in the absence of knowledge of what that value is, we attach a (prior) probability $P(x)$ to every possible value of $x$, where $\sum_{x} P(x)=1$. We shall refer to the function $P(x)$ as the probability distribution of the random variable $x$. The interpretation of probability here is degree of belief in the truth of the proposition that the true dose is $x$. This interpretation provides a mathematical representation of the degree of uncertainty about deterministic quantities: a small bit of probability placed at each of a large number of values of $x$ reflects a high degree of uncertainty, whereas a probability of 1 placed at a single value reflects complete certainty.

We emphasize that the "distribution" $P(x)$ as we have just defined it refers to the distribution of probabilities over all possible values of $x$, which concerns one individual and one exposure period. This is important to note, because we will ultimately be dealing with populations of individuals and exposure periods. The common usage of "distribution" describes the variation in recorded doses for groups of individuals over fixed time periods (e.g. the dose distribution for the 1950 cohort of ORNL workers) and this may cause some confusion. $P(x)$ is not the marginal distribution of true dose in the study cohort.

It is fair to ask why the recorded dose $z$ also needs to be considered a random variable, since it is known. Aside from the clarity that results from starting at the beginning and formally introducing knowledge as it is gained, there is this. For the observed quantity $(z)$ to provide information about the unobserved quantity $(x)$ there must be some postulated relationship between them. Usually this is not deterministic, because of variability in badge readings caused by uncontrollable factors. Instead, the assumed relationship between $z$ and $x$ takes the form of a conditional probability distribution $P(z \mid x)$. (The vertical bar stands for "given".) This is an "if $x$, then $z^{n}$ relationship, but with uncertainty built in, uncertainty that exists prior to the observation of $z$.

We want to arrive at a statement about $x$ given $z$. In the language of proba- 
bility, what is required is the conditional probability distribution $P(x \mid z)$. Bayes' Theorem allows us to get this from $P(z \mid x)$ and $P(x)$ as follows. Define the likelihood function of $x$ given $z$ as

$$
L(x \mid z)=P(z \mid x)
$$

Note that both $P(z \mid x)$ and $L(x \mid z)$ are really the same function of both $x$ and $z$ viewed in two different ways. The distinction is that for a fixed $x$ we have a probability distribution on $z$ (i.e. $\sum_{z} P(z \mid x)=1$ ) and for a fixed $z$ we have a likelihood function of $x$ (i.e. $\sum_{x} L(x \mid z)$ need not be 1). Now, Bayes' Theorem states that

$$
P(x \mid z)=c(z) P(x) L(x \mid z)
$$

where $c(z)$ is a normalizing constant which ensures that $\sum_{x} P(x \mid z)=1$. Both the likelihood (1) and the posterior (conditional) distribution (2) are considered functions of $x$, with $z$ fixed at its observed value.

To implement this approach, $P(z \mid x)$ will be determined by careful consideration of the properties of the measuring device (in this case the film badge and the system used in reading and recording its dose). In effect, $P(z \mid x)$ is the answer to the question: "If the true dose is $x$, what is the probability that the recorded value is $z$ ?" The prior distribution $P(x)$, on the other hand, is considerably more problematic. What beliefs and uncertainties should go into the determination of the prior probabilities? The use of the word "belief" itself connotes a subjectivity that seems inconsistent with true scientific inquiry. In many situations, however, it is possible to formulate a description of $P(x)$ that is acceptably objective.

\subsection{Example}

Here we demonstrate the approach by means of a simple example. Suppose in a given week, the recorded dose to a single film badge is $z=0.4 \mathrm{mSv}$. What is our estimate of the true dose $x$, in the form of the conditional probability distribution $P(x \mid z=0.4)$ ? For $P(x)$, our prior distribution of $x$, we shall use the lognormal 


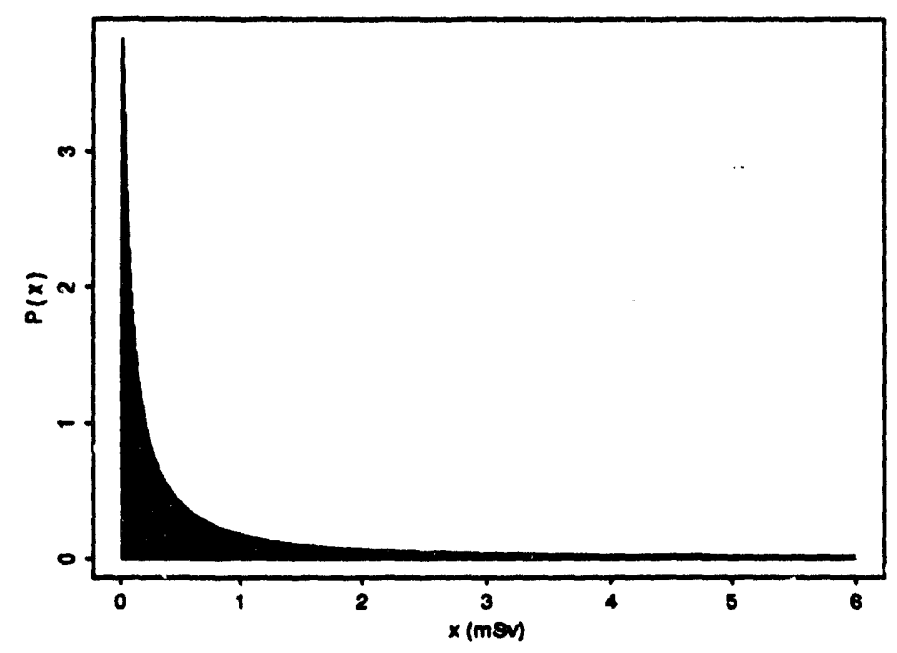

Figure 1: Probability density function for prior distribution of $x$.

distribution. (This means that the natural $\log$ of $x$ has a normal distribution. All logarithms used here will be natural logarithms, i.e., $e^{\log x}=x$.) The lognormal distribution is chosen partly for convenience here, and partly because it reflects the general belief that larger doses are less likely than smaller ones. This belief is consistent with the statistical distribution of film badge readings observed in large populations. Another good choice for the prior would be the gamma distribution, for example.

The lognormal distribution $P(x)$ has parameters $\mu$ and $\sigma$, which are the mean and standard deviation of $\log (x)$. Note also that $\exp (\mu)$ is the median (or 50th percentile) of $x$. In this example we choose a prior with a median of $.3 \mathrm{mSv}$ and 75th percentile of $1 \mathrm{mSv}$. This gives lognormal parameter values of $\mu=-1.204$ and $\sigma=1.821$. These values were chosen so that the distribution agrees reasonably well with a small sample of film badge readings for a single "representative" individual (id142225yr55, see Section 5). Also, it is sometimes suggested that one-tenth of the weekly allowable dose $(.3 \mathrm{mSv})$ was used as a plant action limit. A more rigorous way of using data to specify the prior parameters for a single exposure period is described in Section 4.

The probability density function for this lognormal distribution is shown in Figure 1. The prior probability that the true dose lies between any two values 


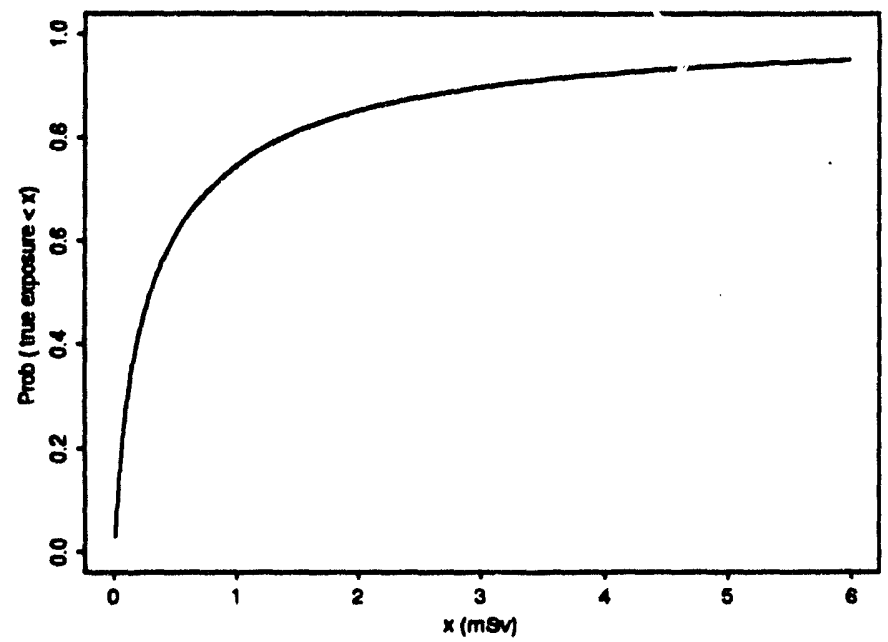

Figure 2: Distribution function for prior distribution of $x$.

is equal to the area under the curve between those values. The area to the left of a given value $x$ is given by the corresponding distribution function in Figure 2. It shows, for a given value $x$, the prior probability that the true dose is less than $x$. The 5th, 25th, 50th, 75th, and 95th percentiles of this distribution are $0.014,0.084,0.30,1.0$, and $6.0 \mathrm{mSv}$, respectively. Note that the spread of the distribution is quite wide, reflecting a considerable amount of prior uncertainty about the true dose $x$ in a given week.

Now we determine $P(z \mid x)$, which is needed to compute the likelihood function. Let $\tilde{z}$ be the expressed dose to the badge; that is, the reading that would be recorded if there were no rounding or censoring. ("Rounding" means that readings are given to the nearest multiple of $0.05 \mathrm{mSv}$. "Censoring" is the practice of recording as zero all readings that are below a certain threshold.) The variability in $\tilde{z}$ for fixed $x$ is intended to represent instrument error and reading error. We shall assume that $\tilde{z}$ has a $\log$ ormal distribution such that $\log (\tilde{z})$ has mean $\log (x)$ and standard deviation $\alpha(x)$, both of which depend on $x$. Thus,

$$
P(\tilde{z} \mid x)=\frac{1}{\sqrt{2 \pi} \tilde{z} \alpha(x)} \exp \left\{\frac{1}{2 \alpha(x)^{2}}[\log (\tilde{z})-\log (x)]^{2}\right\}
$$

At the moment, we are relying mainly on Morgan [14] for information about 
the dependence of $\alpha$ on $x$. :

"... in ordinary routine procedures using technicians to process the film badges, the probable error is about $\pm 30 \mathrm{mrad}$ [or $0.3 \mathrm{mSv}$ of $\gamma$ dose] ... This $\pm 30 \mathrm{mrad}$ does not represent our total probable error in reading the film badiges, except when the readings are from 0 to 30 mrad. If the exposure is to hard $\gamma$-radiation [.1 to $3 \mathrm{MeV}$ ], we can read $100 \mathrm{mrad}$ to $\pm 0.015 \mathrm{rad}$ [i.e., $\pm 15 \%$ ] or $1 \mathrm{rad}$ to $\pm 0.1 \mathrm{rad}$ [i.e., $\pm 10 \%]$."

To translate this information into reasonable values for $\alpha(x)$, we assumed that the "upper 3-standard deviation" limit on $\log (\tilde{z})$ corresponds to the following upper limits on $\tilde{z}: 0.3 \mathrm{mSv}$ at $x=0.01 \mathrm{mSv}, 0.6 \mathrm{mSv}$ at $x=0.3 \mathrm{mSv}, 1.15 \mathrm{mSv}$ at $x=1 \mathrm{mSv}$ and $11 \mathrm{mSv}$ at $\mathrm{x}=10 \mathrm{mSv}$. Under the lognormal assumption, the probability that these upper limits are exceeded is only 0.0013 . That is, we are treating Morgan's (1961) "probable errors" essentially as maximum errors. This interpretation is consistent with Morgan's usage of them to compute the errors for sums of film badge readings. By setting these upper limits to $x e^{3 \alpha}$, i.e., the logarithms of the limits to $\log (x)+3 \alpha$, we find $\alpha=1.134$ at $x=0.01 \mathrm{mSv}$, $\alpha=0.231$ at $x=0.3 \mathrm{mSv}, \alpha=0.0466$ at $x=1 \mathrm{mSv}$, and $\alpha=0.0318$ at $x=10$ $\mathrm{mSv}$. To interpolate between these values, we used a piecewise linear function in $\alpha$ vs. $\log (x)$, which is given in (3). (After trying a log transformation on one or both axes, this was the representation that seemed to give the most regular appearance, in the sense that the slope of the linear pieces is monotone in $x$.)

$$
\alpha(x)= \begin{cases}-0.0884874-0.2654017 * \log (x) & 0<x \leq 0.30 \\ 0.04658731-0.1532109 * \log (x) & 0.30 \leq x \leq 1.00 \\ 0.04658731-0.00643505 * \log (x) & 1.00 \leq x \leq 10.00\end{cases}
$$

The function is plotted in Figure 3.

Because the lognormal distribution is skewed to the right, the "probable error" in the negative direction is less, under our model, than that in the positive direction. For example, the $99 \%$ probability bounds for $\tilde{z}$ are $(0.0005,0.19)$ at 


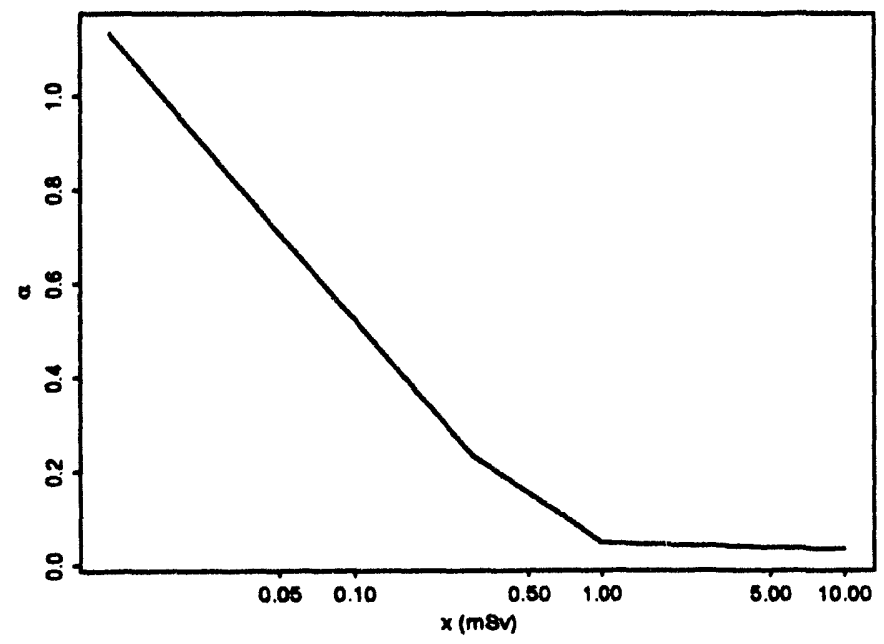

Figure 3: $\alpha$, the standard deviation of $\log (\tilde{z})$ versus true dose.

$x=0.01,(.17,0.54)$ at $x=0.30,(.89,1.13)$ at $x=1.00$, and $(9.21,10.85)$ at $x=10.00$. The bounds based on our interpolation are shown in Figure 4, for $0.01 \leq x \leq 5.00 \mathrm{mSv}$. Figure 5 shows, as an example, $P(\tilde{z} \mid x)$ when $x=0.32$. This is a $\operatorname{lognormal}$ distribution such that $\log (\tilde{z})$ has a normal distribution with mean $\log (.32)=-1.139$ and standard deviation $\alpha(.32)=0.221$ (from (3)).

Recall that $\tilde{z}$ is the expressed dose. We assume in this section that the recorded dose $z$ is obtained from $\tilde{z}$ by rounding to the nearest ${ }^{1}$ multiple of 0.05 $\mathrm{mSv}$, and reporting this value if it is greater than or equal to $0.30 \mathrm{mSv}$. If $\tilde{z}$, after rounding, is less than $0.30 \mathrm{mSv}$, then zero is reported. We call this last policy the censoring convention. These are simplified versions of the rounding and censoring that were done when the historical ORNL data were recorded. For example, the historical data contains a small number of non-zero values that are less than $0.30 \mathrm{mSv}$, which clearly were not censored.

Figure 6 is derived from Figure 5 by applying both the rounding and censoring conventions. This is the assumed distribution of recorded doses that result if the true dose $x=0.32 \mathrm{mSv}$. We can interpret this as a description of the frequency distribution that we would expect if a large number of film badges were exposed

\footnotetext{
${ }^{1}$ We use unequal probability rounding suggested by historical data. Multiples of 0.10 favor multiples of 0.05 at the rate of $85 \%$ to $15 \%$.
} 


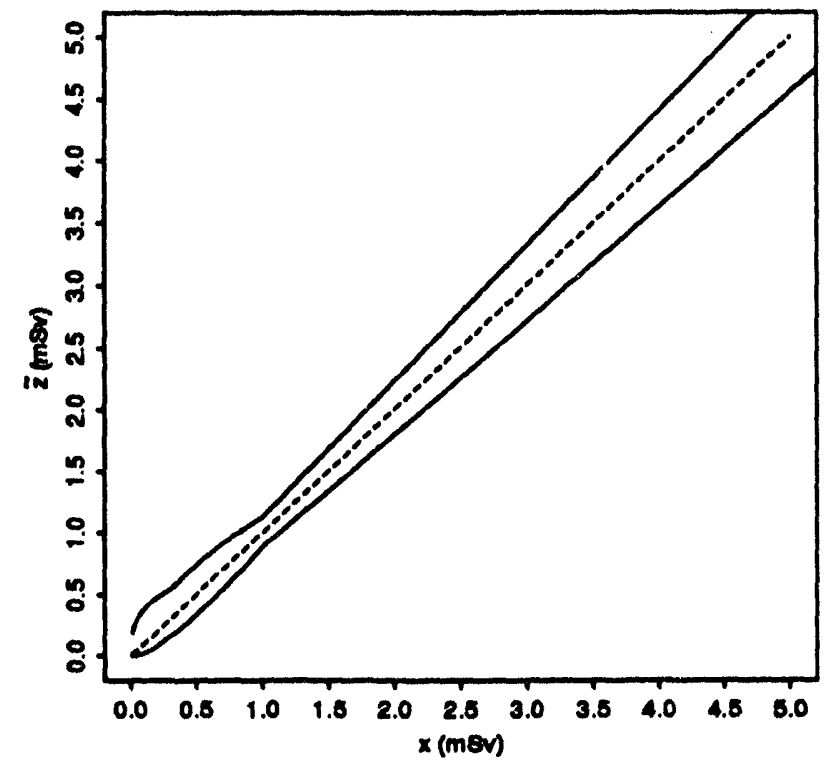

Figure 4: $99 \%$ probability bounds on expressed dose $(\tilde{z})$ versus true dose $(x)$.

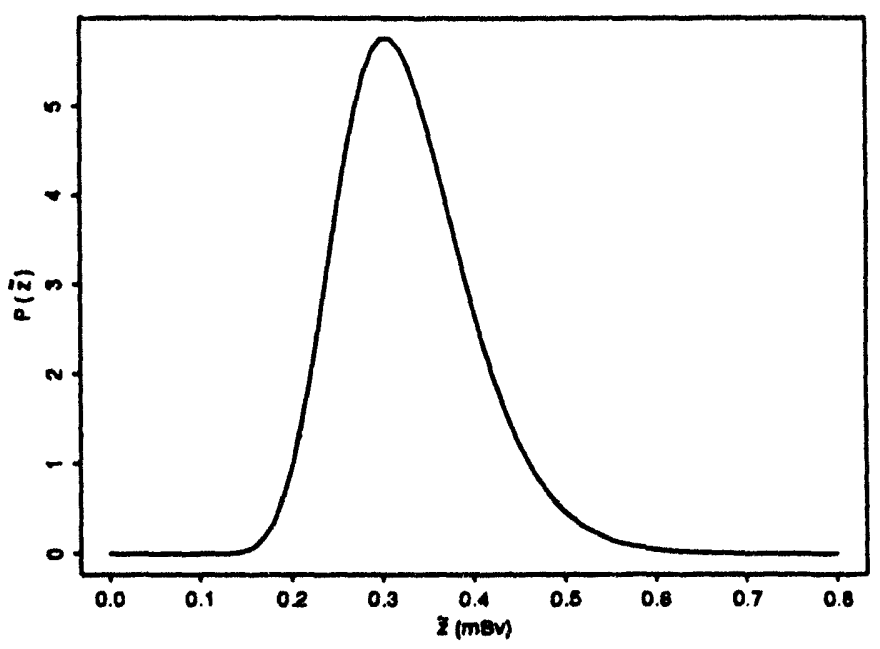

Figure 5: Probability density function of expressed dose $\tilde{z}$ when true dose $x$ is $0.32 \mathrm{mSv}$. 


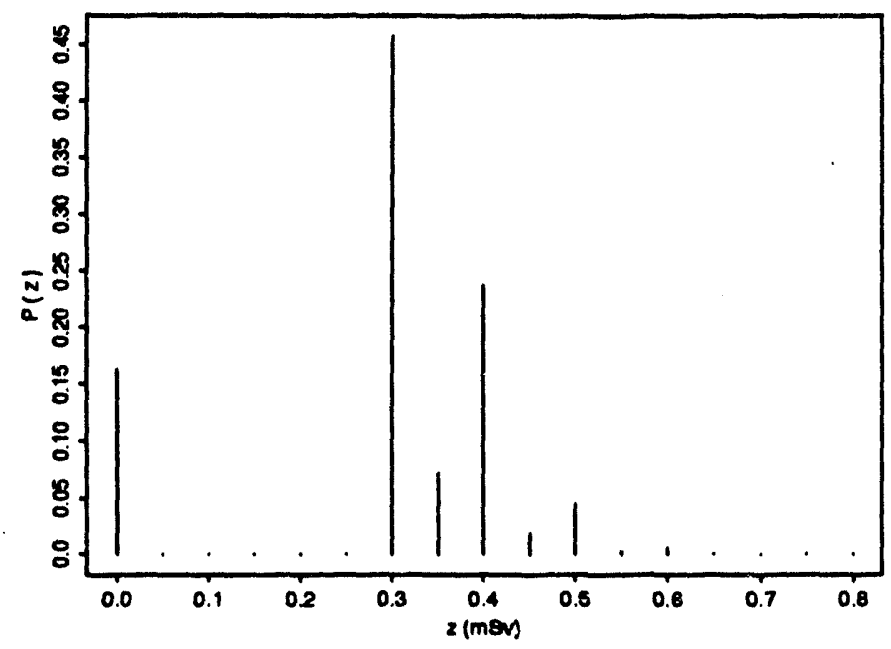

Figure 6: Probability density of recorded dose $z$ when true dose $x$ is $0.32 \mathrm{mSv}$.

to $0.32 \mathrm{mSv}$ and processed through the dosimetry and recording system used prior to 1956.

For fixed $z, L(x \mid z)=P(z \mid x)$ is the likelihood function (1). Figure 7 shows the likelihood function when $z=0.40 \mathrm{mSv}$, the recorded dose to the badge in the present example. This provides an answer to the question: "How likely is it that this observed value $z=0.40 \mathrm{mSv}$ was a result of a possible true dose $x$ ?"

If $z=0.40 \mathrm{mSv}$, then $P(x \mid z)$ is obtained by multiplying $P(x)$ times the likelihood $L(x \mid z=0.40)$ and normalizing so that the sum of probabilities is one (See (2)). This is shown in Figure 8. For computational convenience, we discretized the prior distribution $P(x)$ so that all of the probability lies on multiples of a dose of $0.01 \mathrm{mSv}$ (i.e., $0.00,0.01, .02, \ldots$ ). Thus $P(x)$ is the probability that the lognormal variable with parameters $\mu=-1.204$ and $\sigma=1.821$ lies within 0.005 $\mathrm{mSv}$ of $x$. The posterior distribution shown in Figure 8 necessarily has discrete support also.

Figure 9 compares the posterior distribution shown in Figure 8 with the discretized prior distribution on which the calculation was based. We see that knowledge of the single film badge reading $z=0.40 \mathrm{mSv}$ considerably sharpened our knowledge of $x$, in the sense of reducing the uncertainty about it.

Now suppose the recorded dose $z$ had been zero rather than $0.40 \mathrm{mSv}$. The 


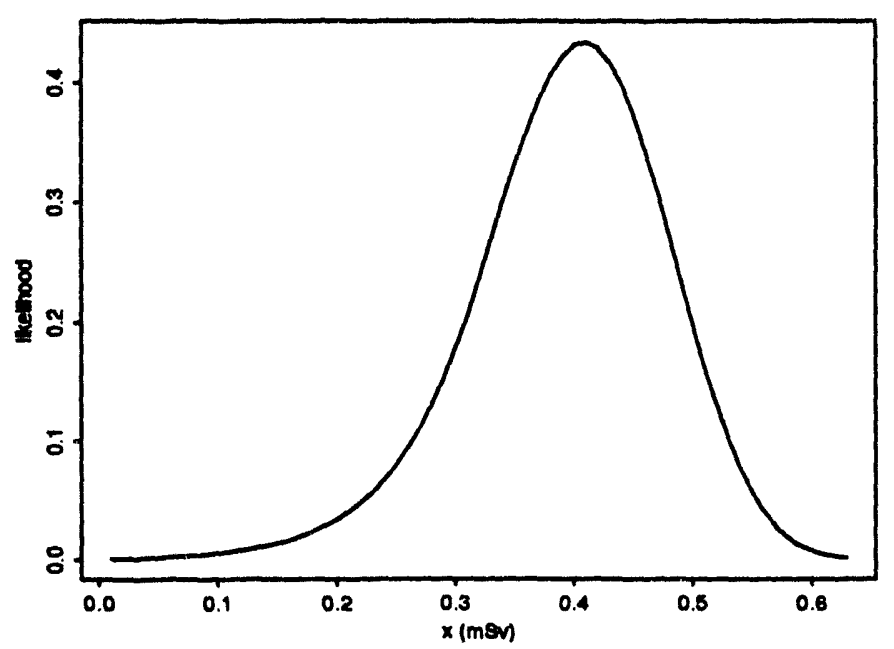

Figure 7: Likelihood of true dose $x$ when recorded dose $z$ is $0.40 \mathrm{mSv}$.

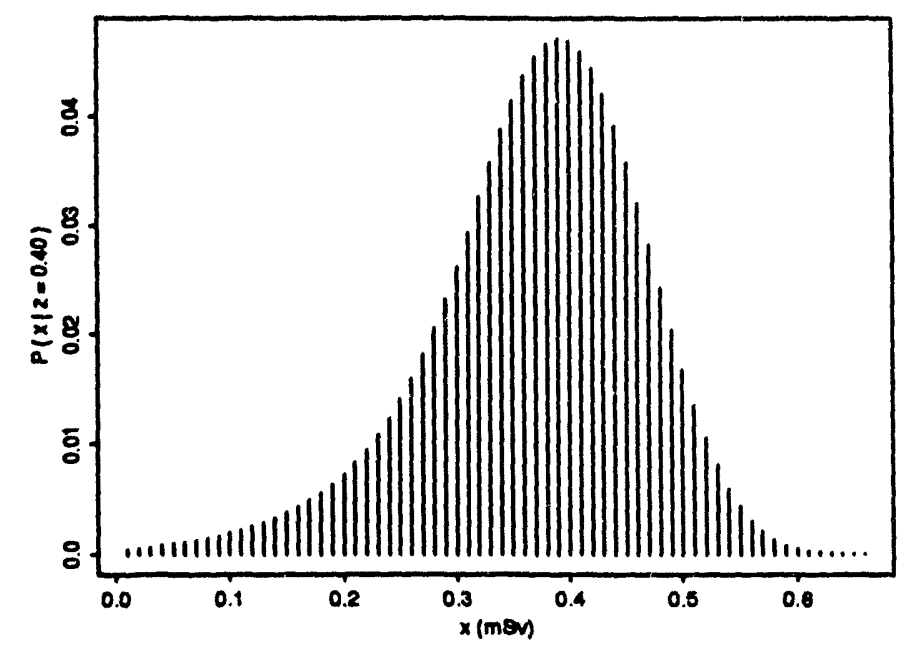

Figure 8: Posterior distribution of true dose $x$ when recorded dose $z$ is $0.40 \mathrm{mSv}$. 


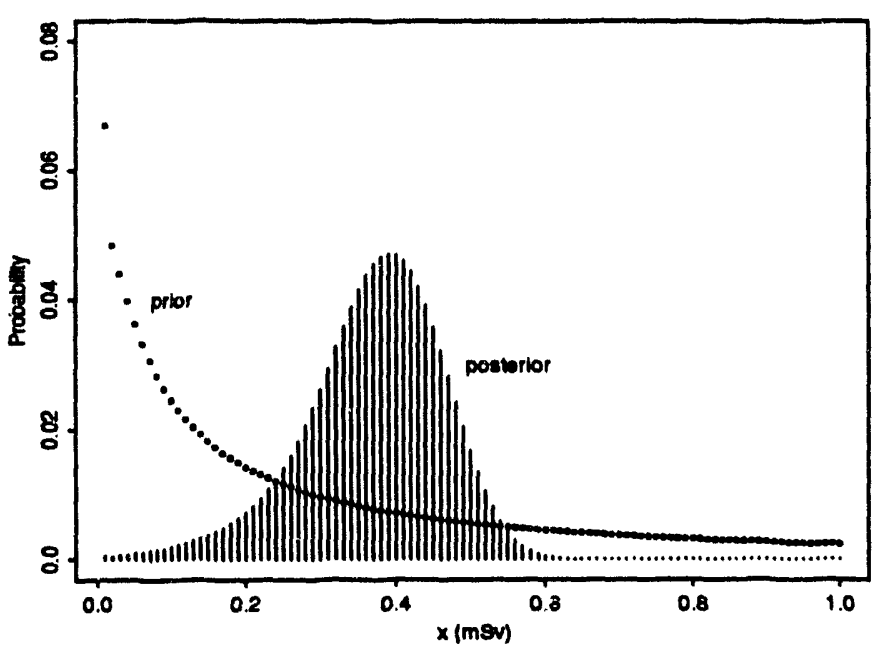

Figure 9: Comparison of prior to posterior distribution of true dose $x$ when recorded dose $z$ is $0.40 \mathrm{mSv}$.

likelihood function in the case $z=0$ is shown in Figure 10 and the posterior distribution is shown in Figure 11. In Figure 12, the prior and posterior distributions are plotted together for comparison. Note that although the film vadge serves to exclude the possibility that the true dose is greater than about $0.4 \mathrm{mSv}$, it does not distinguish well among low values of $x$. In this low dose region, the posterior distribution essentially mirrors the prior distribution, and is quite sensitive to the particular choice of prior.

\section{Estimating Yearly Dose}

We now consider the estimation of a yearly dose based on a sequence of $n$ film badge readings, obtained weekly from a single individual, for example. Let $x_{T}=$ $\sum_{i=1}^{n} x_{i}$ where $x_{i}$ is the true dose to the badge worn by the individual during the $i^{\text {th }}$ week, and let $z_{i}$ be the corresponding recorded dose. We shall denote the set of $n$ true doses by $\mathbf{x}=\left\{x_{1}, x_{2}, \ldots, x_{n}\right\}$, and the set of recorded doses by $\mathrm{z}=\left\{z_{1}, z_{2}, \ldots, z_{n}\right\}$. We want to obtain the posterior distribution $P\left(x_{T} \mid \mathbf{z}\right)$, to serve as an estimate of $x_{T}$. The simplest approach is to treat each week as 


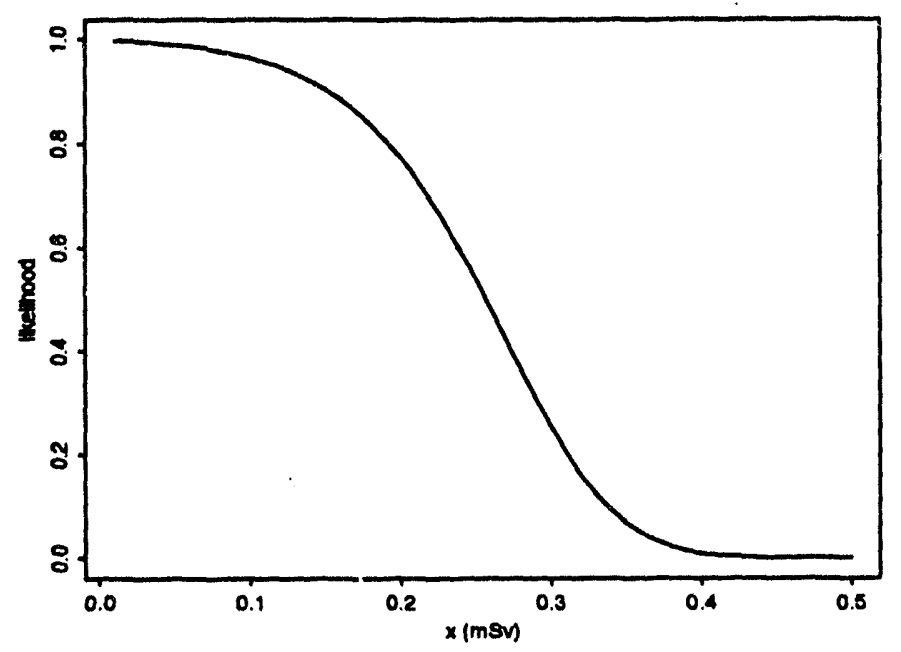

Figure 10: Likelihood of true dose $x$ when recorded dose $z$ is zero.

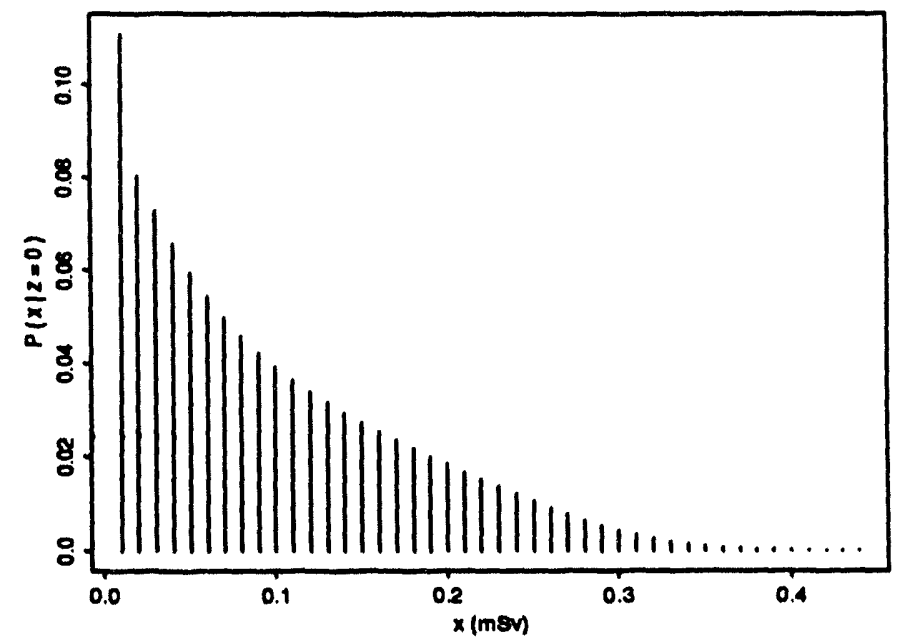

Figure 11: Posterior distribution of true dose $x$ when recorded dose $z$ is zero. 


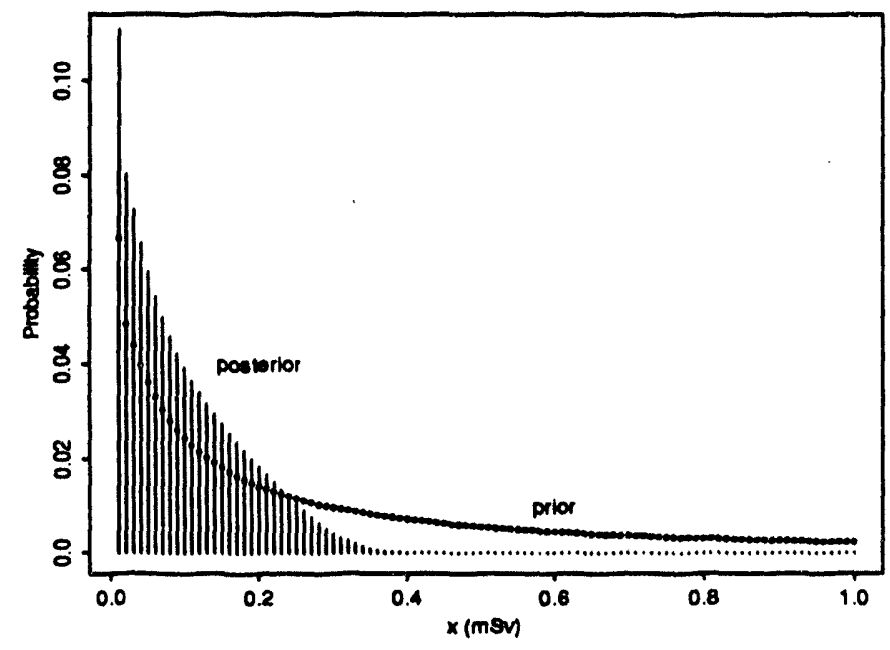

Figure 12: Comparison of prior to posterior distribution of true dose $x$ when recorded dose $z$ is zero.

independent of the others, so that

$$
P(\mathbf{x} \mid \mathbf{z})=\prod_{i=1}^{n} P\left(x_{i} \mid z_{i}\right)
$$

That is, conditional on the set of recorded doses $z$, the set of true doses can be treated as independent random variables, whose individual distributions are given by $P\left(x_{i} \mid z_{i}\right)$. The distribution of the sum $x_{T}$ can be obtained numerically in various ways. Here, we shall simply generate a large random sample from this distribution. To generate $x_{T m}$, the $m$ th value of $x_{T}$ in this sample, we generate first the set $\left\{x_{1 m}, x_{2 m}, \ldots, x_{n m}\right\}$ by drawing $x_{i m}$ from $P\left(x_{i} \mid z_{i}\right)$ for $i=1, \ldots, n$. These values are then summed:

$$
x_{T m}=\sum_{i=1}^{n} x_{i m}
$$

and the procedure is repeated, until a large sample (usually consisting of several thousand values) is generated.

To demonstrate the result of this procedure, we consider weekly records from two individuals. The sequence of recorded film badge readings $(\mathbf{z})$ for person A in 1948 is: 


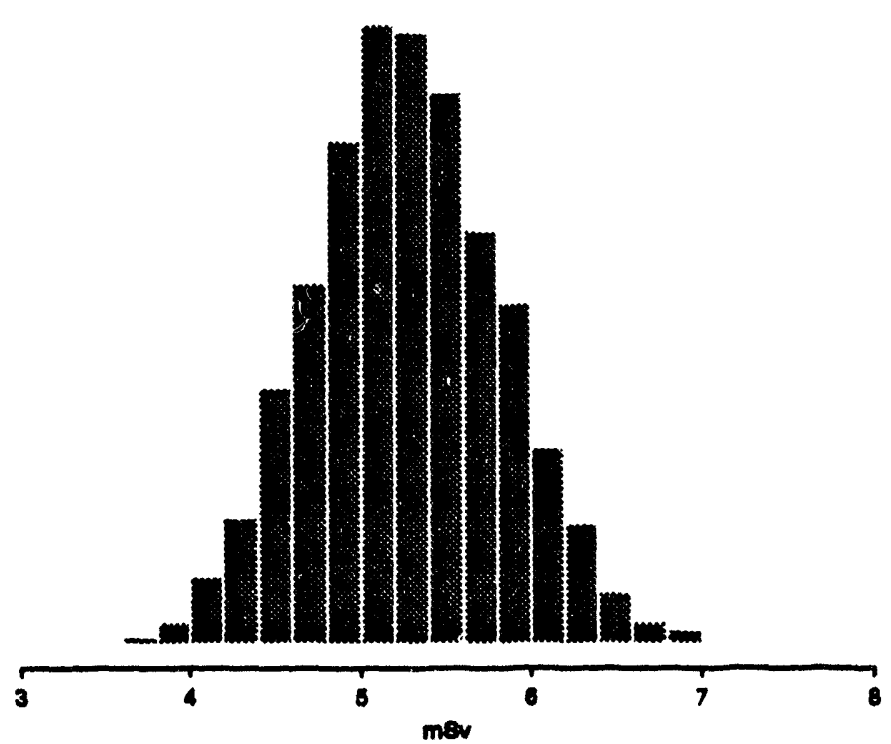

Figure 13: Yearly dose distribution estimate for person A (with recorded dose of $0.65 \mathrm{mSv}$ ) based on a fixed prior that assumes he is a radiation worker with a true weekly dose of about one tenth of the occupational limit.

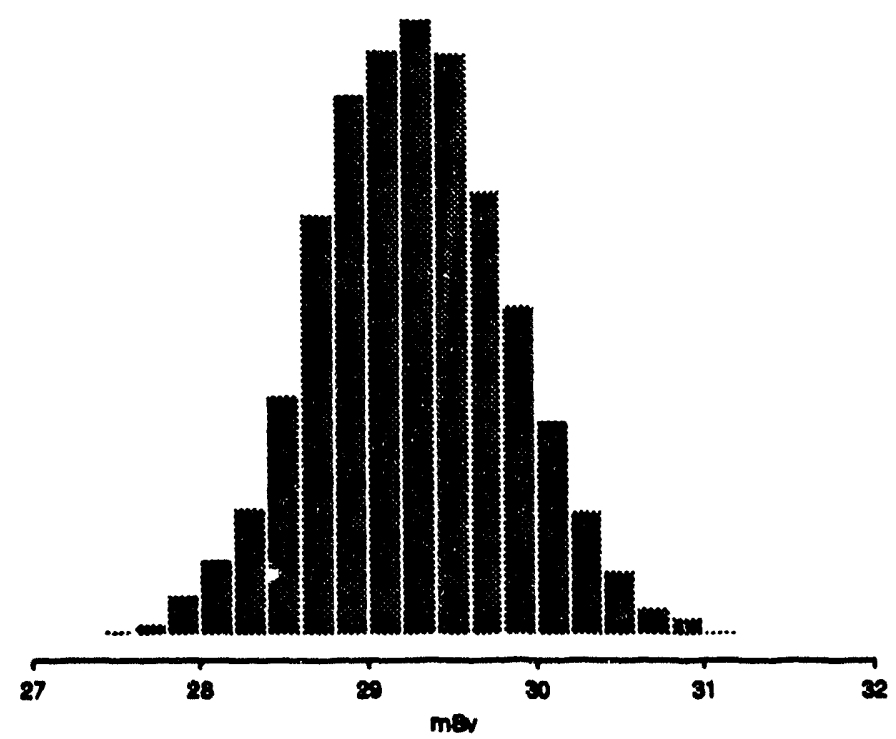

Figure 14: Yearly dose distribution estimates for person $B$ (with recorded dose of $27.2 \mathrm{mSv}$ ) based on a fixed prior that assumes he is a radiation worker with a true weekly dose of about one tenth of tbe occupational limit. 
$\{0,0,0,0,0,0,0,0,0,0,0,0,0,0,0,0,0,0,0,0,0,0,0,0,0,0,0,0,0$, $0,0,0,0,0,0,0,0,0.35,0,0,0,0.30,0,0,0,0,0,0,0,0,0,0\}$,

the total for the year being $0.65 \mathrm{mSv}$.

For each week, $P\left(x_{i} \mid z_{i}\right)$ was computed, in a manner similar to the one we used to generate Figures 8 and 11. (The prior distribution was the same, but the discretization was done in a slightly different way.) Then 5,000 values of $x_{T}$ were generated as we have indicated. A histogram of this sample, which can be viewed as a good approximation to $P\left(x_{T} \mid \mathbf{z}\right)$, is shown in Figure 13. This suggests that the true 1948 dose of person $A$ is roughly between 3.5 and $7 \mathrm{mSv}$.

For person $\mathrm{B}$, the sequence of recorded badge readings in 1954 is:

$$
\begin{aligned}
& \{2.1,0,0.8,0.95,1.4,0.5,0,0.7,0,1.4,0.3,3.45,1,1.6,0,1.6,0,0.8,0.5, \\
& 0.7,0,0.4,0,0,0.6,0.75,0,0,0,0,0,0,0,0,1.5,0,0,0,0,0,0.9,0.7,1.1, \\
& 0,0,0.9,0,0,0,1.7,0.55,0.3\}
\end{aligned}
$$

the total for the year being $27.2 \mathrm{mSv}$. Figure 14 shows our approximation to $P\left(x_{T} \mid \mathbf{z}\right)$ for this individual. It suggests that the true 1954 dose for person $B$ is roughly between 27.5 and $31 \mathrm{mSv}$.

Note that for both individuals our estimate of the yearly dose is centered higher than the sum of the recorded doses. This is more pronounced with the lower recorded annual dose.

\section{Use of Other Data}

The available weekly data (described in Section 5) also includes a pocket-meter dose corresponding to each recorded film-badge dose. This weekly pocket-meter dose was calculated as the sum of minimum daily readings of a pair of pocket meters. We will refer to this weekly sum as the weekly pocket-meter dose and denote it by $w$. Also, let $w=\left\{w_{1}, w_{2}, \ldots, w_{n}\right\}$ be the vector of $n$ weekly pocketmeter doses that corresponds to $\mathrm{z}$, the vector of recorded film-badge doses.

Here we assume that the weekly pocket-meter dose, $w$, is correlated with the true dose, 2 , to the film badge and consider it only as additional informa- 


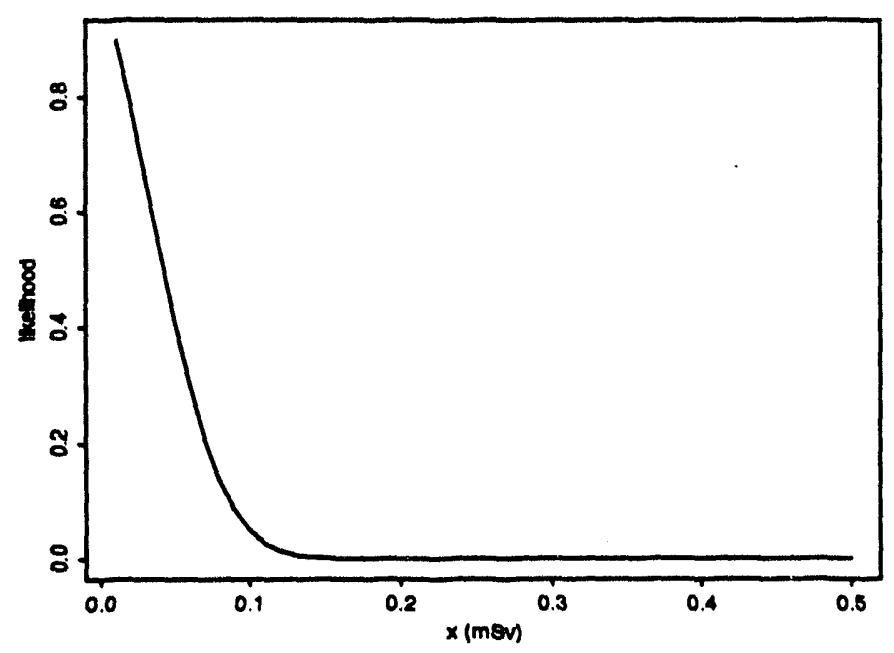

Figure 15: Likelihood of true dose $x$ when recorded dose $z$ is zero without censoring.

tion to specify our prior and likelihood functions. A more rigorous approach to the inclusion of pocket-meter data would account for error and bias of the daily pocket-meter dose measurement system in a manner similar to that used in previous sections for the recorded film badge dose. This more rigorous approach will be discussed in a separate report.

\subsection{Modifying the Likelihood}

The likelihood function for $z=0$, shown in Figure 10 is based on the censoring convention of recording as zero any reading that would be rounded below 0.3 $\mathrm{mSv}$. When the weekly pocket-meter dose $w$ is zero, it is reasonable to conclude for $z=0$ that the expressed dose $\tilde{z}$ was within rounding error of zero and no censoring was necessary. Figure 15 shows the likelihood function of $x$, the true dose, when $z=0$ and no censoring is performed. We will refer to this as the "rounded" zero likelihood and to the likelihood of Figure 10 as the "censored" zero likelihood. The prior and posterior distributions of true dose $x$ that result from the "rounded" zero likelihood are in Figure 16. When we compare Figure 16 to Figure 12, we see that the "rounded" zero likelihood puts considerably more posterior mass near zero, effectively excluding dose above $0.15 \mathrm{mSv}$. 


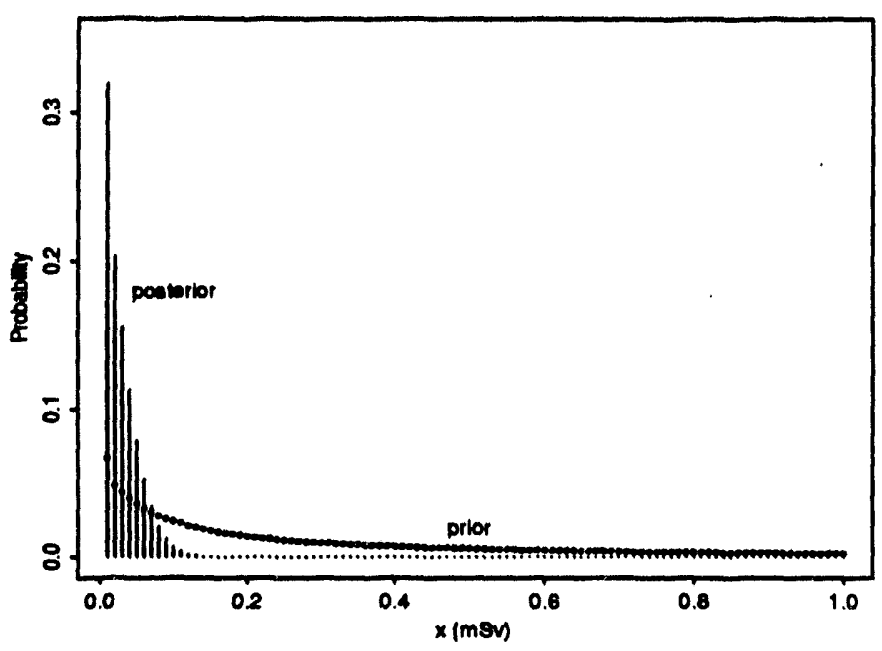

Figure 16: Comparison of prior and posterior distributions of true dose $x$ when recorded dose $z$ is zero without censoring.

We demonstrate the effect of the "rounded" zero likelihood on $P\left(x_{T} \mid \mathbf{z}\right)$ of persons $A$ and $B$ introduced in Section 3. The sequence of weekly pocket-meter doses $w$ for person $A$ is:

$\{0,0,0.05,0,0,0,0,0,0,0,0,0,0,0,0,0,0,0,0,0,0,0,0.1,0,0,0,0$, $0,0,0,0,0,0,0.05,0,0,0,0,0.05,0,0,0,0,0,0,0,0,0,0,0,0,0\}$,

and the same sequence for person $B$ is:

$$
\begin{aligned}
& \left\{\begin{array}{l}
0.6,0.15,0.85,0.8,0.55,0,0.5,0,0.15,0.6,0.55,4.3,1.3,1.7,1.95,0.9, \\
0.45,1.4,1.6,0.4,0.1,0.3,0,0.05,0.7,0,0.1,0.1,0,0,0.15,0,0,0.05,1.7, \\
0.1,0,0,0,0.15,0.35,0.1,0.1,0.7,0.45,0.2,0,0,0,0.35,0.2,0.65\}
\end{array}\right.
\end{aligned}
$$

Their yearly dose distributions are shown in Figures 17 and 18. As expected, both distributions have shifted closer to the recorded dose, although both are still centered considerabiy higher than the recorded dose. The shift toward reccrded dose is greater for person A, because of a larger proportion of "rounded" zeros. Rounded zeros also have less uncertainty and this results in a narrower distribution for person A. 


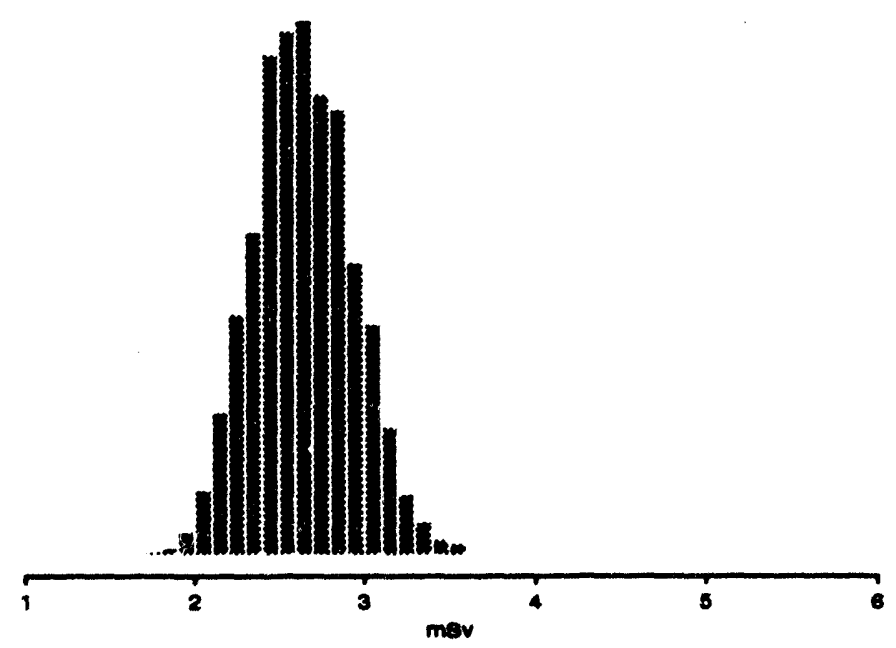

Figure 17: Yearly dose distribution estimate for person A (with recorded dose of $0.65 \mathrm{mSv}$ ) allowing "rounded" zeros and with a fixed prior.

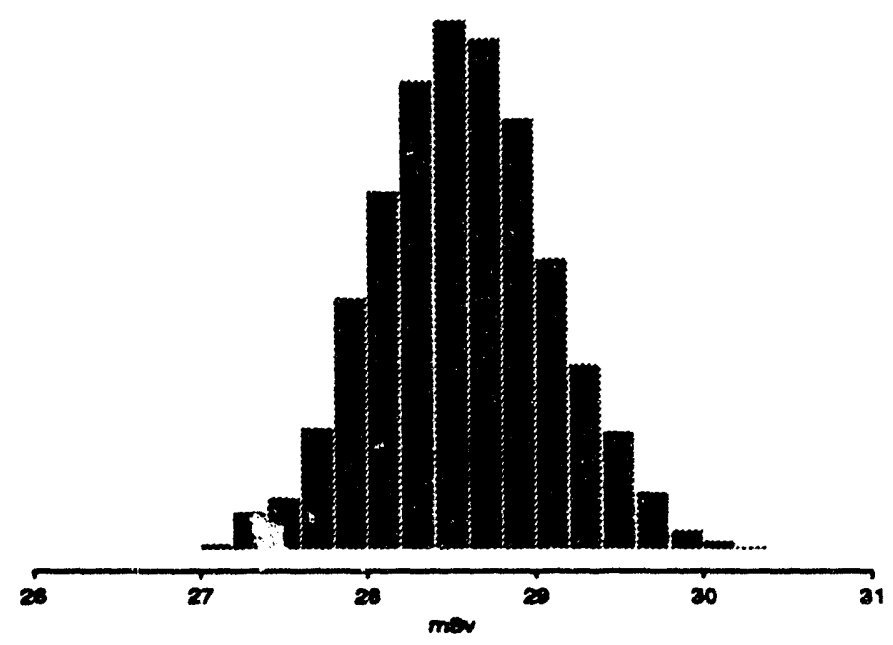

Figure 18: Yearly dose distribution estimate for person B (with recorded dose of $27.2 \mathrm{mSv}$ ) allowing "rounded" zeros and with a fixed prior. 


\subsection{Specifying the Prior}

In our examples to this point, the parameters $\mu$ and $\sigma \rightarrow f$ the prior distribution $P(x)$ have been fixed. To lessen the chance of being misled by poor specification of these parameters, we will use pocket-meter data to specify $\mu$ and $\sigma$. In particular, the natural logarithm of the weekly pocket-meter dose will be used as $\mu$. The parameter $\sigma$ will be set to a value that puts the 95th percentile of $P(x)$ at $\mu+6.00$ $\mathrm{mSv}$. This ensures that the prior puts a nontrivial probability on values as high as $6.00 \mathrm{mSv}$ regardless of the value of $w$. This provides a large amount of prior uncertainty and allows the recorded film badge dose to be the dominating factor that determines the posterior dose distribution.

Some weekly pocket-meter doses are missing. In such cases we use that individual's weekly pocket-meter dose average over the weeks that it is not missing. When all weekly pocket-meter doses are missing, we consider them as zero for the purpose of specifying the prior. (See paragraph below for special treatment of zeros.) Here we implicitly assume that an individual with no pocket meter readings is not a radiation worker.

The use of the lognormal distribution as our prior on $x$, the true dose to the film badge, implicitly assumes that $x>0$. This is consistent with the belief that the true dose may be very small but can never be zero. Since the pocket meters have a sensitivity threshold, many weekly pocket-meter doses are recorded as zero. Because we cannot take the logarithm of zero, the accepted practice is to choose a small positive value $c_{0}$ to replace the zero. We use the special case of $z=0$ and $\mathbf{w}=0$ to calibrate the value. This is where results are most sensitive to the choice of $c_{0}$. The value $c_{0}=0.0003 \mathrm{mSv}$ puts the 95 th percentile of $P\left(x_{T} \mid \mathrm{z}\right)$ at $0.30 \mathrm{mSv}$. That is, we choose $c_{0}$ so that the probability is .95 that the yearly true dose is below $0.30 \mathrm{mSv}$, when all pocket-meter and film-badge doses are zero. This distribution is shown in Figure 19. As the recorded dose increases, sensitivity of the true dose distribution to the choice of $c_{0}$ decreases. For example, the mean of the true dose distribution for an individual with a recorded yearly dose of $16.75 \mathrm{mSv}$ (which consists of about $50 \%$ weekly zeros) 


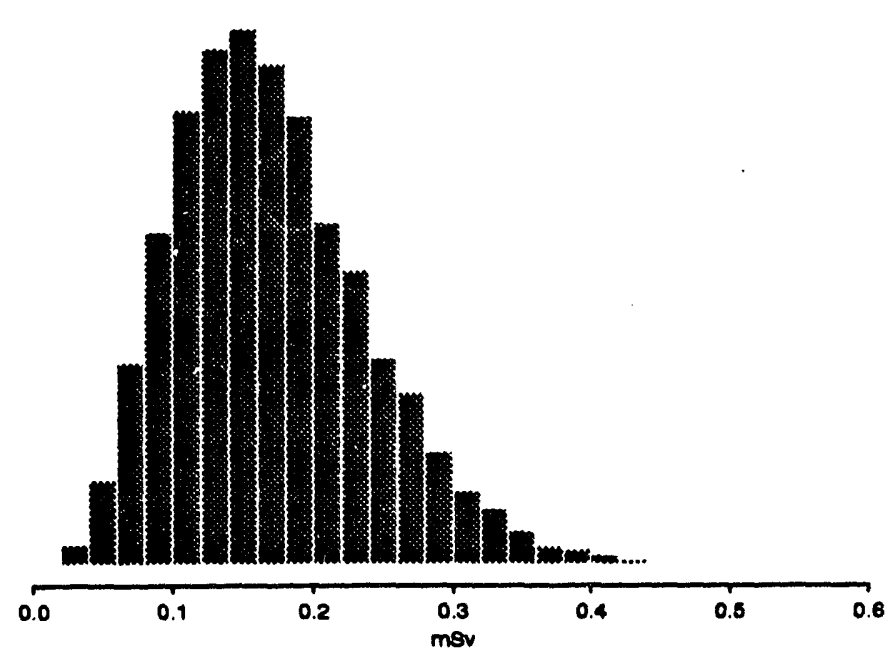

Figure 19: Yearly dose distribution estimate for an individual with zero recorded film-badge dose and all zero weekly pocket-meter doses.

increases by $0.004 \mathrm{mSv}$ in response to doubling the $c_{0}$ value. This is a very small change, particularly when we consider that $c_{0}$ is a weekly quantity and the dose distribution is a yearly quantity.

Again, we demonstrate the the effect of specifying the prior parameters $\mu$ and $\sigma$ with pocket-meter data on $P\left(x_{T} \mid \mathrm{z}\right)$ of persons $\mathrm{A}$ and $\mathrm{B}$. These are shown in Figures 20 and 21. Both distribution have again shifted closer to the recorded dose.

\section{Application to ORNL Cohort}

To illustrate the dose estimation method that we have developed, a sample of hard copy records was obtained from the ORNL dosimetry files. The hard copy records contain the detailed daily and weekly monitoring results for each "personyear" selected. The sample was obtained in two stages. It includes a stratified random sample of exposed workers (150 person-years) with yearly film badge totals that are greater than zero. The remainder of the sample (100 person-years) was obtained by sampling at random from all person-year records over the period from 1945-1955 when film badges were evaluated on a weekly basis. The weekly film-badge dose and the pocket-meter dose were abstracted from the hard copy 


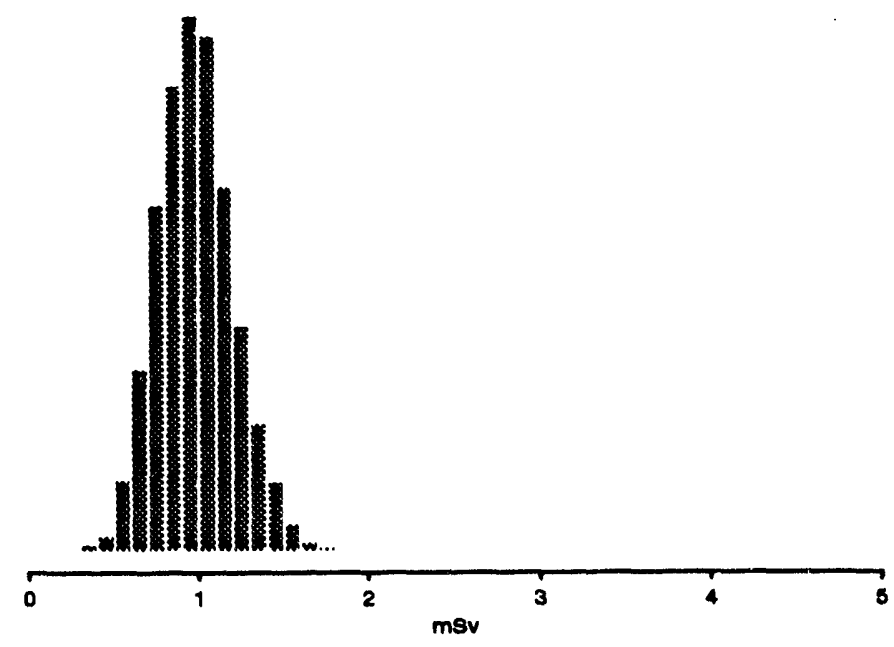

Figure 20: Yearly dose distribution estimate for person A (with recorded dose of $0.65 \mathrm{mSv}$ ) allowing "rounded" zeros and with pocket-meter sp cified priors.

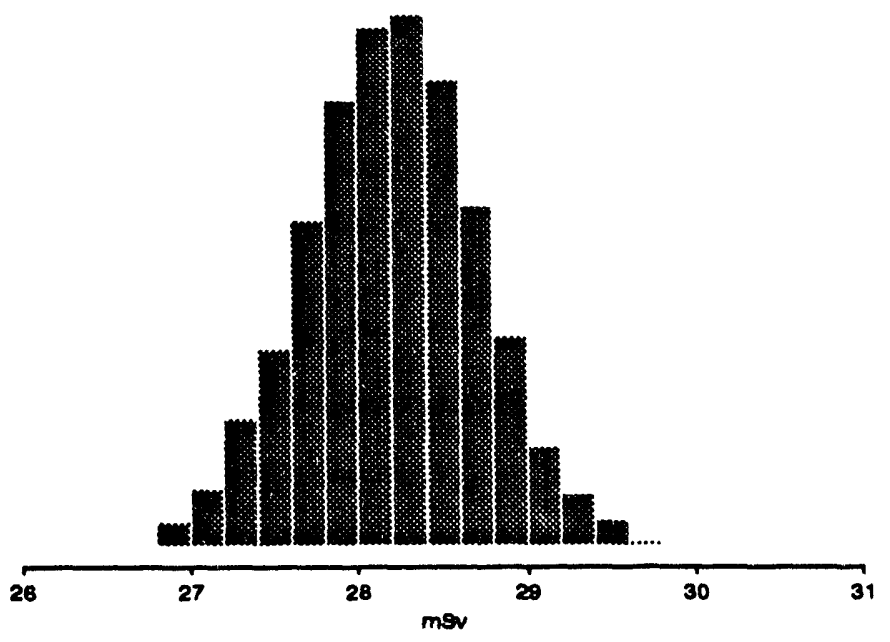

Figure 21: Yearly dose distribution estimate for person $B$ (with recorded dose of $27.2 \mathrm{mSv}$ ) allowing "rounded" zeros and with pocket-meter specified priors. 
records. This data has also been used to develop a preliminary dose-adjustment procedure in another study (see [4] for further details). It is important to note that the data currently being used in epidemiologic studies of ORNL workers $[2,8,22,23,24]$ consists of the yearly total of the weekly film-badge readings for each worker. The detailed weekly records are not available in machine readable form at this time.

\subsection{Dose Estimation}

This section describes the results of applying our dose estimation procedure to the 150 person-years (out of the possible 250 described above) that had at least thirty weekly records. The results are summarized in Figures 22-25, which show boxplots of $P(\mathbf{x} \mid \mathbf{z})$ for each person-year. The boxplots show the 1, 25, 50, 75, and 99 percentiles of each distribution. In addition a bold glyph indicates the relative position of the recorded dose for each person-year. The person-years along the vertical axis are in increasing order of recorded dose. We use the available pocketmeter data to modify priors and likelihoods as discussed in Section 4. As we pointed out in Section 2.2, the data contain a few non-zero recorded film-badge doses below the censoring point of $0.30 \mathrm{mSv}$. We use the "rounded" likelihood in each of these cases, because clearly no censoring was performed.

We make the following observations about Figures 22-25. In making some of these observations we use our knowledge of the underlying weekly dose data for each person-year.

- Almost all distributions are centered above the recorded film-badge dose. Thus recorded doses systematically underestimate the true dose.

- The recorded dose is below the one percentile of the distribution in about half the cases. This indicates a severe underestimate of the true dose by the recorded dose.

- The most severe underestimate occurs for person-years that have many zero recorded film-badge doses which correspond to non-zero pocket-meter 


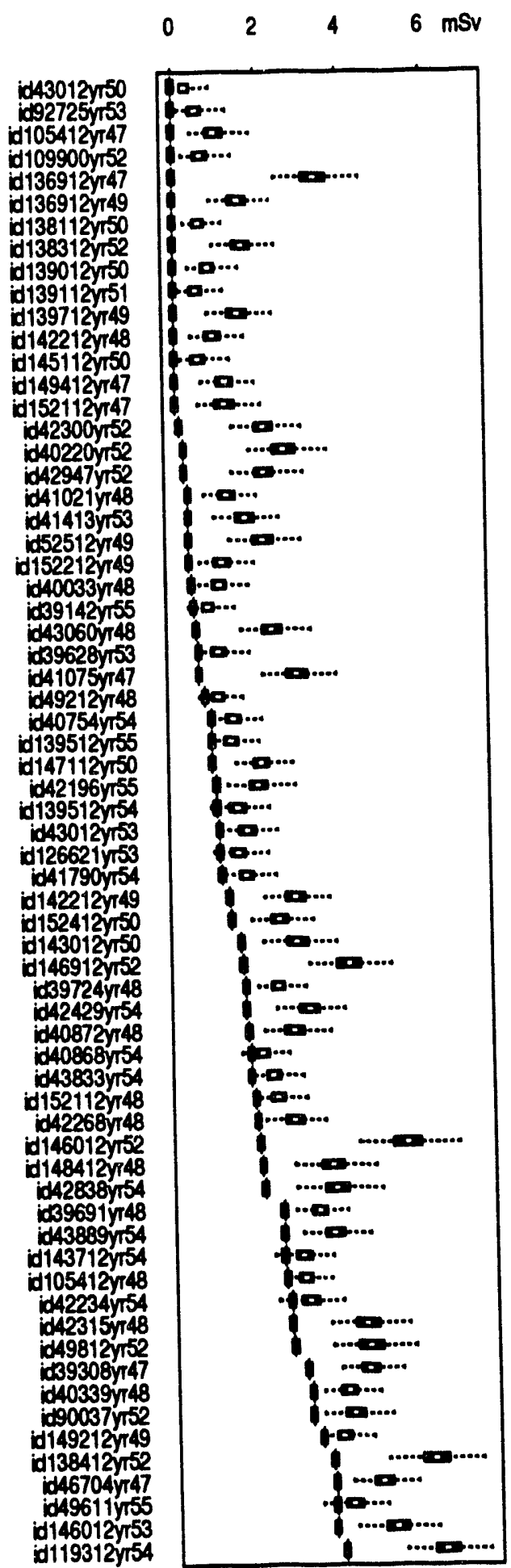

Figure 22: Boxplots of yearly dose distribution estimates and the corresponding recorded dose $(\mathrm{mSv})$ for a sample of the ORNL cohort. 


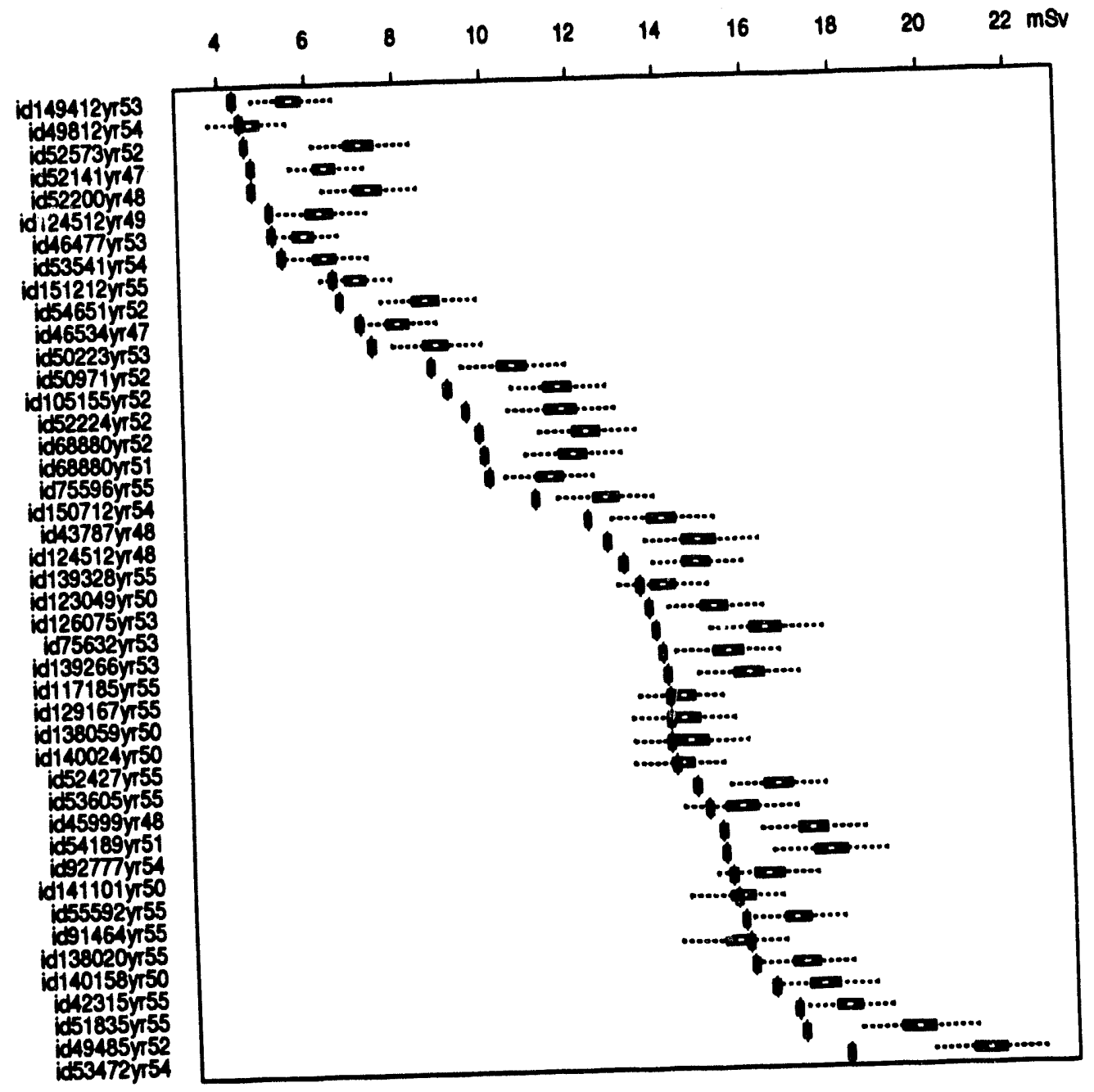

Figure 23: Boxplots of yearly dose distribution estimates and the corresponding recorded dose (mSv) for a sample of the ORNL cohort (continued). 


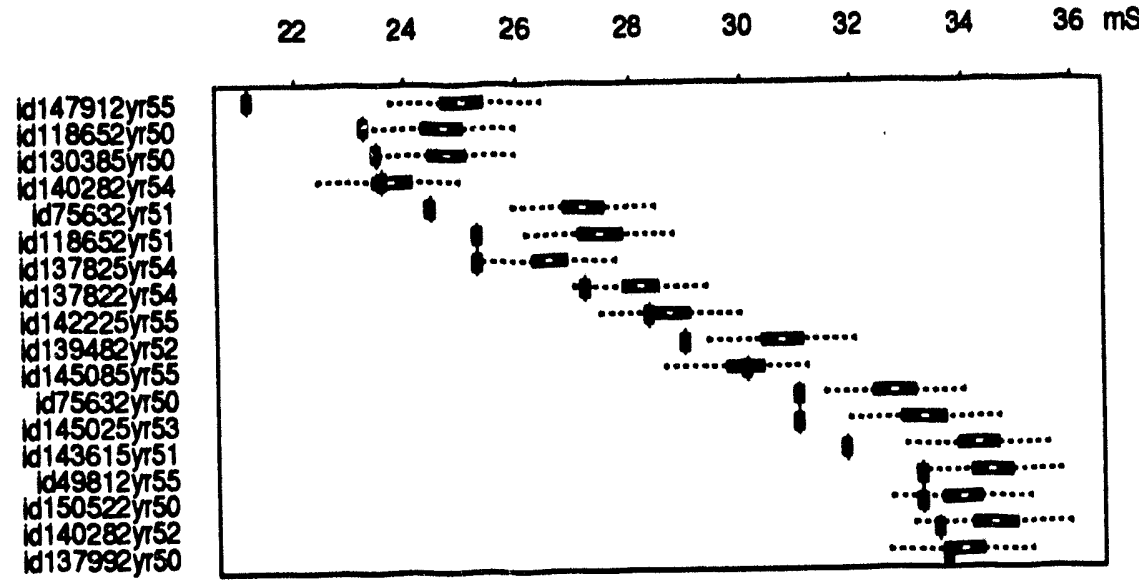

Figure 24: Boxplots of yearly dose distribution estimates and the corresponding recorded dose (mSv) for a sample of the ORNL cohort (continued).

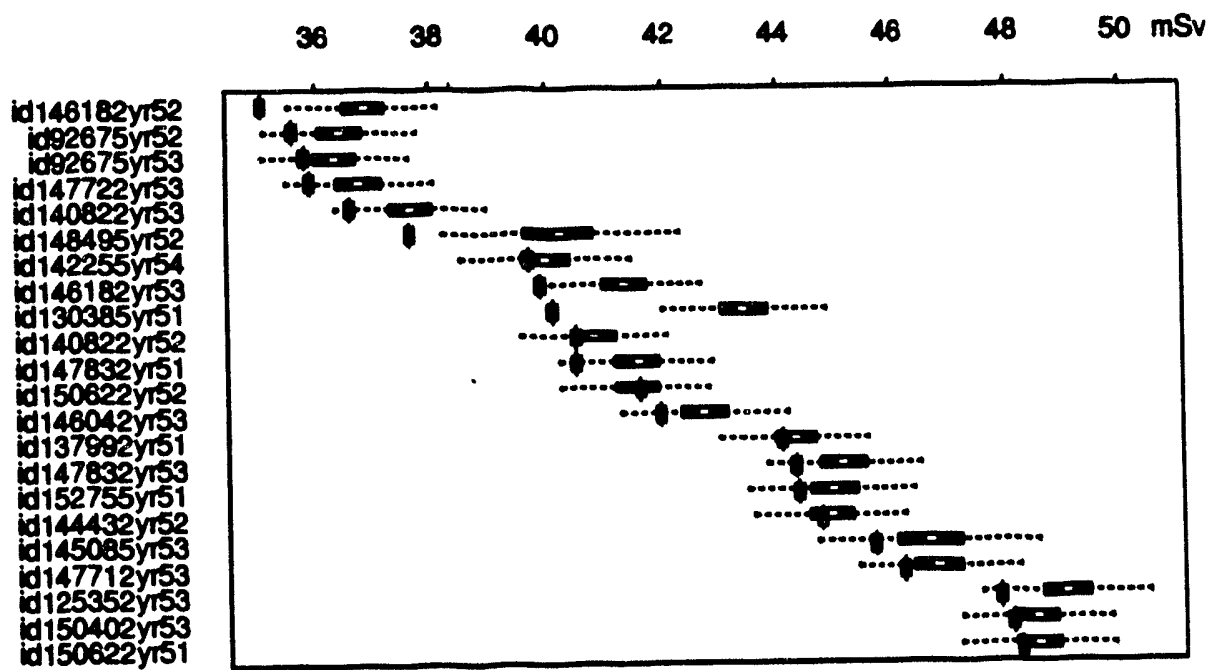

Figure 25: Boxplots of yearly dose distribution estimates and the corresponding recorded dose (mSv) for a sample of the ORNL cohort (continued). 
doses.

- The relative uncertainty is greatest at lower recorded dose levels.

- The large differences in uncertainty at the lower recorded dose levels appear to be mostly due differences in content of "rounded" and "censored" zeros. Censored zeros (a non-zero weekly pocket-meter dose corresponding to a zero recorded film-badge dose) introduce more uncertainty than rounded zeros (a zero film-badge dose corresponding to a zero recorded pocket-meter dose).

\subsection{Dose-Response Analysis}

The results in this report indicate that dose estimates obtained for ORNL workers before 1956 that, are currently being used in epidemiologic studies contain a large systematic negative bias. It is also apparent that there is considerable uncertainty in theses dose estimates that should be taken into account when they are used in dose-response studies of radiation effects. Studies published to date $[8,22,24]$ that involve ORNL workers are based on the recorded film badge doses and have not taken the uncertainties described here into account in the statistical analysis. A general approach to statistical inference that reflects measurement uncertainty has been described by [3] and discussed further in the context of radioepidemiologic studies by [7]. In this setting of chronic disease epidemiology, the relation between time to failure and covariates that change over time is often described with a relative risk regression model [17], e. g.

$$
\lambda[t \mid x(t)]=\lambda_{0}(t) \theta[x(t) ; \beta]
$$

which represents the probability of disease incidence per unit time for persons at age $t$ with covariate history $x(t)$ (i.e. $x(t)$ is the true dose history up to time $t)$. The two forms of the relative risk function $\theta[x(t) ; \beta]$ that are of particular 
interest are the loglinear relative risk regression mode]

$$
\log \{\theta[x(t) ; \beta]\}=x^{*}(t) \beta,
$$

and the linear relative risk regression model

$$
\theta[x(t) ; \beta]=1+x^{*}(t) \beta
$$

where $x^{*}(t)$ is a known function of the covariate history which is usually taken to be the lagged cumulative lifetime dose.

In this report we have proposed a model that describes the effect of "measurement error", i.e. a model that relates the recorded dose $z$ to the underlying true dose $x$ for an individual during a specified period of time. The statistical problem that remains is to develop a method for estimating the dose-response parameter given the time to failure and the dose history distributions $P(x(t) \mid z(t))$ for each individual in the cohort. The practical problem that remains for the ORNL cohort is to obtain the historical daily pocket meter and weekly film badge data in electronic form so that valid dose estimates can be obtained for subsequent statistical analysis.

\subsection{Computational Issues}

The computational requirements of the method developed in this report are substantial. In this section we present only a broad outline. Details will be discussed in a separate report.

The continuous distributions $P(x), P(z \mid x)$, and $P(x \mid z)$ are each represented with a discrete approximation on 500 points. Since the lognormal distribution is used in both $P(x)$ and $P(z \mid x)$, the computation of $P(x \mid z)$ is performed on a log scale rather than the linear scale, thus avoiding the evaluation of many logs. This and many other ideas are used to reduce the computational burden.

The yearly dose distribution $P\left(x_{T} \mid \mathbf{z}\right)$ can be computed in various ways. We chose to compute it by generating a large random sample from it. There are faster 
ways (such as convolution via the discrete fast Fourier transform) to compute this distribution, but our choice of the simulation method was made in part because of its simplicity and in part because it easily extends to the method of Gibbs sampling (see [6]), which we plan to use in some future extensions of this work.

All of the methods to compute $P\left(x_{T} \mid \mathbf{z}\right)$ require computing $P\left(x_{i} \mid z_{i}\right)$ for each week $i$ of a person-year. We exploit some storage-computation tradeoffs here, which are available because of frequent repetition of some recorded film-badge and pocket-meter doses. As we outlined in Section 3, to generate a sample point from $P\left(x_{T} \mid \mathbf{z}\right)$, we draw a point from each of $P\left(x_{i} \mid z_{i}\right)$ and report the sum.

A current high performance workstation (we used an IBM RS/6000) can generate 5,000 sample points from $P\left(x_{T} \mid \mathbf{z}\right)$ in about two minutes. This results in roughly five hours of computation for the entire set of 150 person-years used in this report. This computation was performed several times, in order to experiment with the various prior and likelihood specifications described in Section 4. To make this a more interactive task, we prepared a parallel implementation of our software. The parallel implementation utilizes PVM software [5] on a cluster of high performance workstations and reduce the five-hour computational task to about half an hour.

Expanding this computation to the entire ORNL cohort, after it is available in machine readable form, will require even closer attention to computational issues. The use of individual dose-distribution data in dose-response parameter estimation will likely also be based on simulation and thus require state-of-the-art computational resources.

\section{Summary}

We have developed methodology to account for uncertainty and bias in measurements of individual occupational radiation dose using weekly dosimetry data. The product of this methodology is an estimate of the true dose for a person-year in the form of a probability distribution.

The key component of this methodology is a model of the proced"res used 
in a single film badge measurement. This is the likelihood function. We have attempted to formulate a realistic model that includes the most important aspects of film-badge dose measurement in the 1940s and 1950s, however the historical information is often sketchy. Perhaps more importantly, we have shown that the model can easily incorporate much detail and as more information becomes available this model can be modified.

Another important component is the prior distribution whose importance grows with uncertainty in measurement. Since the largest uncertainty in recorded doses results from censoring, dose estimates from data containing many censored zeros are sensitive to prior specification.

We have shown how other data can be used to influence the prior and the likelihood. We have used pocket-meter data to specify the prior location and also to indicate whether a film-badge zero resulted from only rounding or from rounding and censoring. A more rigorous way of including pocket-meter data that accounts for bias and measurement error will be discussed in a separate report. Potentially additional data, such as occupation or work location, can be used to modify the prior and the likelihood.

We applied our methodology to a sample of dose histories obtained from hard copy dosimetry records at Oak Ridge National Laboratory (ORNL). The estimated dose distributions show that recorded doses generally have a strong negative bias. The bias is present at all yearly dose levels, but it is most severe at low to medium dose levels. This raises serious questions about the validity of the historical personnel dosimetry data that is currently being used in low-dose studies of nuclear industry workers. In particular, the results in this report indicate that ORNL workers employed prior to 1957 are likely to have had doses that were higher than those recorded. Consequently, the dose-respon:e coefficients that are based directly on the recorded doses $[8,22,24]$ are probably biased and their uncertainty is understated. 


\section{Acknowledgments}

We thank the Office of Radiation Protection at ORNL for providing the data used in this study, and the Center for Epidemiologic Research at Oak Ridge Institute for Science and Education for help with data entry and verification. We also thank Asim Yar Khan of the University of Tennessee for his help in implementing some of the software.

\section{References}

[1] A. C. Brown and C. B. MacDonald, editors. The Secret History of the Atomic Bomb. Dial Press/James Wade, New York, 1977.

[2] E. Cardis and J. Estéve. Uncertainties in recorded doses in the nuclear industry: identification, quantification and implications for epidemiological studies. Radiat. Prot. Dosim., 36(2/4):315-319, 1991.

[3] David Clayton. Models for the longitudinal analysis of cohort and casecontrol studies with inaccurately measured exposures. In James H. Dwyer, Manning Feinleib, Peter Lippert, and Hans Hoffmeister, editors, Statistical Models for Longitudinal Studies of Health, volume 16 of Monographs in Epidemiology and Biostatistics, pages 301-331. Oxford University Press, 1992.

[4] E. L. Frome, D. L. Cragle, J. Watkins, S. Wing, C. Shy, W. Tankersley, and C. M. West. A mortality study of employees of the nuclear industry in Oak Ridge, Tennessee. Technical Report ORNL TM-12275, Oak Ridge National Laboratory, Oak Ridge, TN 37831, 1993.

[5] G. A. Geist and V. S. Sunderam. Network-based concurrent computing on the PVM system. Concurrency: Practice \& Experience, 4(4):293-311, 1992.

[6] Alan E. Gelfand and Adrian F. M. Smith. Sampling-based approaches to calculating marginal densities. J. Amer. Statist. Assoc., 85:398-409, 1990. 
[7] E. S. Gilbert. Accounting for bias and uncertainty resulting from dose measurement errors and other factors. Technical Report PNL-SA-18907, Pacific Northwest Laboratory, Richland, WA 99352, February 1990.

[8] E. S. Gilbert, S. A. Fry, L. D. Wiggs, G. L. Volez, D. L. Cragle, and G. R. Petersen. Analyses of combined mortality data on workers at the Hanford Site, Oak Ridge National Laboratory, and Rocky Flats Nuclear Weapons Plant. Radiat. Res., 120:19-35, 1989.

[9] J. C. Hart. A progress report dealing with the derivation of dose data from ORNL personnel exposure records applicable to the Mancuso study. Technical Report ORNL/M-2614 (formerly, ORNL CFM 66-1-84), Oak Ridge National Laboratory, Oak Ridge, TN, 37831, January 141966.

[10] G. D. Kerr, R. H. Ritchie, S. V. Kaye, and J. S. Wassom. History of the Health and Safety Research Division at Oak Ridge National Laboratory. Technical Report ORNL/M-2108, Oak Ridge National Laboratory, Oak Ridge, TN, 1992.

[11] George D. Kerr. Missing dose from mortality studies of radiation effects among workers at Oak Ridge National Laboratory. Health Phys., in press, 1993.

[12] G. W. Kneale, T. Sorahan, and A. M. Stewart. Evidence of biased recording of radiation doses of Hanford workers. Am. J. Ind. Med., 20:799-803, 1991.

[13] Fred C. Maienschein and R. W. Peelle. Radiation dosage estimation and health risk. JAMA, 267:929, 1992.

[14] K. Z. Morgan. Dosimetry requirements for protection from ionizing radiation. In Selected Topics in Radiation Dosimetry, pages 3-23. Vienna: International Atomic Energy Agency, IAEA Proceedings Series, 1961. 
[15] K. Z. Morgan. Techniques of personnel monitoring and radiation surveying. In A. H. Snell, editor, Nuclear Instruments and Their Uses, volume 1, pages 391-469. John Wiley \& Sons, 1962.

[16] K. D. Nichols. The Road to Trinity: A Personal Account of How America's Nuclear Policies Were Made. William Morrow and Company, New York, 1987.

[17] R. L. Prentice. Covariate measurement errors and parameter estimation in a failure time regression model. Biometrika, 69(2):331-342, 1982.

[18] James S. Press. Bayesian Statistics: Principles, Models, and Applications. John Wiley \& Sons, New York, 1989.

[19] Daniel J. Strom. Estimating individual and collective doses to groups with 'less than detectable' doses: A method for use in epidemiologic studies. Health Phys., 51(4):437-445, 1986.

[20] N. A. Taylor. Estimation of dose received when dosemeter results are recorded below a threshold level. J. Radiol. Prot., 11(3):191-198, 1991.

[21] R. H. Wilson, W. V. Baumgartncr, J. J. Fix, and L. L. Nichols. Description and evaluation of the hanford personnel dosimeter program from 1944 to 1989. Technical Report PNL-7447, Pacific Northwest Laboratory, Richland, WA, 1990.

[22] S. Wing, C. M. Shy, J. L. Wood, S. Wolf, D. L. Cagle, and E. L. Frome. Mortality among workers at Oak Ridge National Laboratory: Evidence of radiation effects in follow-up through 1984. JAMA, 265:1397-1402, 1992.

[23] S. Wing, C. M. Shy, J. L. Wood, S. Wolf, and D. L. Cragle. Reply to comments by Maienschein and Peelle. JAMA, 267:929-930, 1992.

[24] Steve Wing, Carl M. Shy, Joy L. Wood', Susanne Wolf, Donna L. Cragle, William Tankersley, and E. L. Frome. Job factors, radiation and cancer 
-36 -

mortality at Oak Ridge National Laboratory: Follow-up through 1984. Am. J. Ind. Med., 23:265-275, 1993. 
ORNL-6778

\section{INTERNAL DISTRIBUTION}

1. A. B. Ahmed

2. B. R. Appleton

3. B. A. Berven

4. J. S. Bogard

5. E. Brackett

6. T. S. Darland

7. E. Dixon

8. D. J. Downing

9. K. F. Eckerman

10-14. E. L. Frome

15. S. Garrett

16. R. N. Hamm

17. F. Hartman

18. J. B. Hunt

19-23. G. D. Kerr

24. C. A. Little

25. G. L. Love

26. P. Y. Lu

27. J. C. Miller

28. M. D. Morris
29. C. E. Oliver

30-34. G. Ostrouchov

35-39. Stephanie Raby

40. D. E. Reichle

41. P. S. Rohwer

42. J. C. Ryman

43. C. S. Sims

44-48. R. F. Sincovec

49. R. E. Swaja

50. J. H. Swanks

51. C. C. Travis

52-56. R. C. Ward

57. J. K. Williams

58. Central Research Library

59. K-25 Applied Technology Library

60-61. Laboratory Records Dep.

62. Laboratory Records - RC

63. Patent Office

64. Y-12 Technical Library

\section{EXTERNAL DISTRIBUTION}

65. S. Abrahamson, Director of Research, Radiation Effects Research Foundation, 5-2 Hijiyama Park, Minami-ku, Hiroshima, 732 Japan

66. J. A. Auxier, Auxier \& Associates, Inc., 111 Mabry Hood Road, Suite 500, Knoxville, TN 37922

67. J. R. Beall, Office of Health and Environmental Research, ER-72, U. S. Department of Energy, Washington, DC 20585

68. Don E. Bihl, Battelle-Northwest Laboratory, P. O. Box 999, Richland, WA 99352

69. J. D. Boice, Radiation Epidemiology Branch, National Cancer Institute, Executive Plaza North, Room 408, Bethesda, MD 20892

70. Richard J. Brake, Los Alamos National Laboratory, MS-K483, Los Alamos, NM 87545

71. N. Breslow, Department of Biostatistics, University of Washington, Seattle, WA 98195

72. Roger W. Brockett, Pierce Hall, 29 Oxford Street, Harvard Unive:sity, Cambridge, Mn 02138

73. A. Brodsky, 2765 Ocean Pines, \#27 St. Martins Lane, Berlin, MD 21811-9127 
74. B. G. Brooks, Epidemiology and Health Surveillance, EH-42 CC 270, U. S. Department of Energy, 19901 Germantown Road, Germantown, MD 20874

75. E. Cardis, International Agency for Research on Cancer, 150 Cours Albert Thomas, 69372 Lyon Cedex 08, France

76. Harvey Checkoway, Department of Environmental Health, University of Washington, SC34, Seattle, WA 98195

77. G. Cowper, 4 Cartier Circle, Deep River, Ontario, Canada K0J 1P0

78. D. L. Cragle, Medical Division, Oak Ridge Institute for Science and Education, P. O. Box 117, Oak Ridge, TN 37831

79. K. W. Crase, Westinghouse Savannah River Co., Savannah River Site, Bldg. 735A, Aiken SC 29802

80. D. Crawford-Brown, Department of Environmental Science, University of North Carolina, Chapel Hill, NC 27599-7400

81. Owen Devine, Radistion Studies Branch, Center for Disease Control, 1600 Clifton Road NE, Mailstop F-28, Atlanta, GA 30333

82. Donald J. Dudziak, Department of Nuclear Engineering, 110B Burlington Engineering Labs, North Carolina State University, Raleigh, NC 27695-7909

83. Mark Durst, Lawrence Berkeley Laboratory, 1 Cyclotron Road, Building 50, Room 3238, Berkeley, CA 94720

84. J. Estéve, International Agency for Research on Cancer, 150 Cours Albert Thomas, 69372 Lyon Cedex 08, France

85. Roger B. Falk, EG\&G, Rocky Flats, P. O. Box 464, Golden, CO 80401

86. J. J. Fix, Battelle Pacific Northwest Laboratories, Battelle Boulevard, P.O. Box 999, Richland, WA 99352

87. D. Flanders, Department ot Epidemiology, School of Public Health, Emory University, Atlanta, GA 30322

88. Jerome Friedman, Department of Statistics, Sequoia Hall, Stanford University, Stanford, CA 94305

89. R. J. M. Fry, Radiation Research, Biology Division, ORNL, P.O.Box 2009, Oak Ridge, TN 37831-8077

90. S. A. Fry, Medical Division, Oak Ridge Institute for Science and Education, P. O. Box 117, Oak Ridge, TN 37831

91. Warren Galke, Occupational Medicine Group, Los Alamos National Laboratory HSE-2, MS: K404, Los Alamos, New Mexico 87545

92. E. S. Gilbert, Battelle Pacific Northwest Laboratories, Battelle Boulevard, P.O. Box 999, Richland, WA 99352

93. M. Ginevan, Step 5 Corporation, 1101 17th Street NW, Suite 501, Washington, DC 20036

94. R. Goldsmith, Epidemiology and Health Surveillance, EH-42 CC 270, U. S. Department of Energy, 19901 Germantown Road, Germantown, MD 20874 
95. Peter Groer, University of Tennessee, Department of Nuclear Engineering, 315 Pasqua Engineering Bldg., Knoxville, Tennessee 37996-2300

96. N. H. Harley, New York University, Department of Epidemiology, 550 First Avenue, New York, NY 10016

97. Dan Hitchcock, Office of Scientific Computing, ER-7, Applied Mathematical Sciences, Office of Energy Research, U.S. Department of Energy, Washington, DC 20585

98. R. W. Hornung, National Institute for Occupational Safety and Health, 4676 Columbia Parkway, Cincinnati, OH 45226-1998

99. G. R. Howe, NCIC Epidemiology Unit, University of Toronto, McMurrich Puilding, 12 Queens Park Crescent W, Toronto, Ontario, Canada M5S 1A8

100. Fred Howes, Office of Scientific Computing, ER-7, Applied Mathematical Sciences, Office of Energy Research, U.S. Department of Energy, Washington, DC 20585

101. S. Jablon, Radiation Epidemiology Branch, Division of Cancer Etiology, National Cancer Institute, 6130 Executive Boulevard, EPN 408, Bethesda, MD 20852

102. S. V. Kaye, Analysas Corporation, 151 LaFayette Drive, Oak Ridge, TN 37830

103. G. M. Kendall, National Radiological Protection Board, Chilton, Didcot, Oxon OX11 ORQ, United Kingdom

104. G. W. Kneale, Department of Public Health and Epidemiology, University of Birmingham, Edgbaston, Birmingham B15 2TT, United Kingdom

105. C. E. Land, Radiation Epidemiology Branch, Division of Cancer Etiology, National Cancer Institute, 6130 Executive Boulevard, EPN 404, Bethesda, MD 20852

106. James E. Leiss, Route \#2, Box 142C, Broadway, VA 22815

107. A. C. Lucas, Victoreen, Inc., 6000 Cochran Road, Cleveland, OH 44139-3395

108. B. H. Margolin, Department of Biostatistics, University of North Carolina, School of Public Health, Chapel Hill, NC 27599-7400

109. G. Matanoski, Johns Hopkins University, 624 North Broadway, Baltimore, MD 21205

110. Fred C. Maienschein, 838 W Outer Drive, Oak Ridge, TN 37831

111. C. B. Meinhold, Radiological Sciences Division, Bldg. 703 M, Brookhaven National Laboratory, Long Island, NY 11937

112. Ted Meinhardt, Deputy Directory, Division of Surveillance, Hazards Evaluation \& Field Studies, R-12, National Institute of Occupational Health, 4676 Columbia Parkway, Cincinnati, OH 45226

113. Neville Moray, Department of Mechanical and Industrial Engineering, University of Illinois, 1206 West Green Street, Urbana, IL 61801

114. K. Z. Morgan, 106 Connors Drive, Oak Ridge, TN 37830

115. William E. Murray, National Institute for Occupational Safety and Health 4676 Columbia Parkway Cincinnati, Ohio 45226

116. David Nelson, Director of Office of Scientific Computing, ER-7, Applied Mathematical Sciences, Office of Energy Research, U.S. Department of Energy, Washington, DC 20585 
117. Robert W. Peele, 130 Oklahoma Avenue, Oak Ridge, TN 37831

118. H. J. Pettengill, Epidemiology and Health Surveillance, EH-42 CC 270, U. S. Department of Energy, 19901 Germantown Road, Germantown, MD 20874

119. D. A. Pierce, Department of Statistics, Radiation Effects Research Foundation, 5-2 Hijiyama Park, Minami-ku, Hiroshima, 732 Japan

120. J. W. Poston, Department of Nuclear Engineering, T'exas A\&M University, College Station, TX 77843

121. D. L. Preston, Department of Statistics, Radiation Effects Research Foundation, 5-2 Hijiyama Park, Minami-ku, Hiroshima, 732 Japan

122. Ross L. Prentice, Fred Hutchinson Cancer Research Center, 1124 Columbia Street, Seattle, WA 98104

123. Carl Quong, Lawrence Berkeley Laboratory, 1 Cyclotron Road, Building 50, Berkeley, California 94720

124. Robert Rinsky, National Institute for Occupational Safety and Health, 4676 Columbia Parkway, Cincinnati, Ohio 45226

125. Jerome Sacks, National Institute of Statistical Sciences, P.O. Box 14162, Research Triangle Park, NC 27709-4162

126. D. M. Schaeffer, Radiation Policy Division, Defense Nuclear Agency, 6801 Telegraph Road, Alexandria, VA 22310-3398

127. Scott Schneider, Occupational Health Foundation, 1126 16th Street NW, Washington, DC 20036

128. W. J. Schull, Epidemiological Research Center, School of Public Health, University of Texas Health Science Center, Houston, TX 77225

129. L. R. Shenton, Office of Computing and Information Service, Boyd Graduate Studies Building, University of Georgia, Athens, GA 30602

130. R. E. Shore, Institute of Environmental Medicine, New York University, 341 East 25th Street, 2nd Floor, New York, NY 10010-2598

131. W. K. Sinclair, National Council on Radiation Protection, 7910 Woodmont Avenue, Suite 800, Bethesda, MD 20814

132. J. Smith, Radiation Studies Branch, Center for Disease Control, 1600 Clifton Road, NE, Mailstop F-28, Atlanta, GA 30333

133. Daniel L. Solomon, Department of Statistics, North Carolina State University, P.O. Box 5457, Raleigh, NC 27650

134. H. B. Spitz, University of Cincinnati, Department of Mechanical, Industrial and Nuclear Engineering, Cincinnati, OH 45221-0072

135. A. M. Stewart, Department of Public Health and Epidemiology, University of Birmingham, Edgbaston, Birmingham B15 2TT, United Kingdom

136. H. Stockwell, Epidemiology and Health Surveillance, EH-42 CC 270, U. S. Department of Energy, 19901 Germantown Road, Germantown, MD 20874

137. C. H. Strader, Epidemiology and Health Surveillance, EH-42 CC 270, U. S. Department of Energy, 19901 Germantown Road, Germantown, MD 20874 
138. Werner Stuetzle, Department of Statistics, GN-22, University of Washington, Seattle, WA 98195

139. W. G. Tankersley, Oak Ridge Institute of Science and Education, Center for Epidemiological Research, P.O. Box 117, Oak Ridge, TN 37831-0117

140. D. Thomas, Department of Preventive Medicine, University of South California, 1420 San Pablo Street, PMB B201, Los Angelcs, CA 90033-9987

141. M. Vaeth, Department of Statistics, Radiation Effects Research Foundation, 5-2 Hijiyama Park, Minami-ku, Hiroshima, 732 Japan

142. Ray A. Waller, S-1, Statistics, Los Alamos National Laboratory, P.O. Box 1663, Los Alamos, NM 87545

143. J. Watkins, Medical Division, Oak Ridge Institute for Science and Education, P. O. Box 117, Oak Ridge, TN 37831

144. J. E. Watson, School of Public Health, University of North Carolina, Chapel Hill, NC 27599-7400

145. C. M. West, Oak Ridge Institute of Science and Education, Center for Epidemiological Research, P.O. Box 117, Oak Ridge, TN 37831-0117

146. S. Wing, Department of Epidemiology, School of Public Health, University of North Carolina, Chapel Hill, NC 27599-7400

147. Office of Assistant Manager for Energy Research and Development, U.S. Department of Energy, Oak Ridge Operations Office, P.O. Box 2001, Oak Ridge, TN 37831-8600

148-149. Office of Scientific and Technical Information, P.O. Box 62, Oak Ridge, TN 37830 


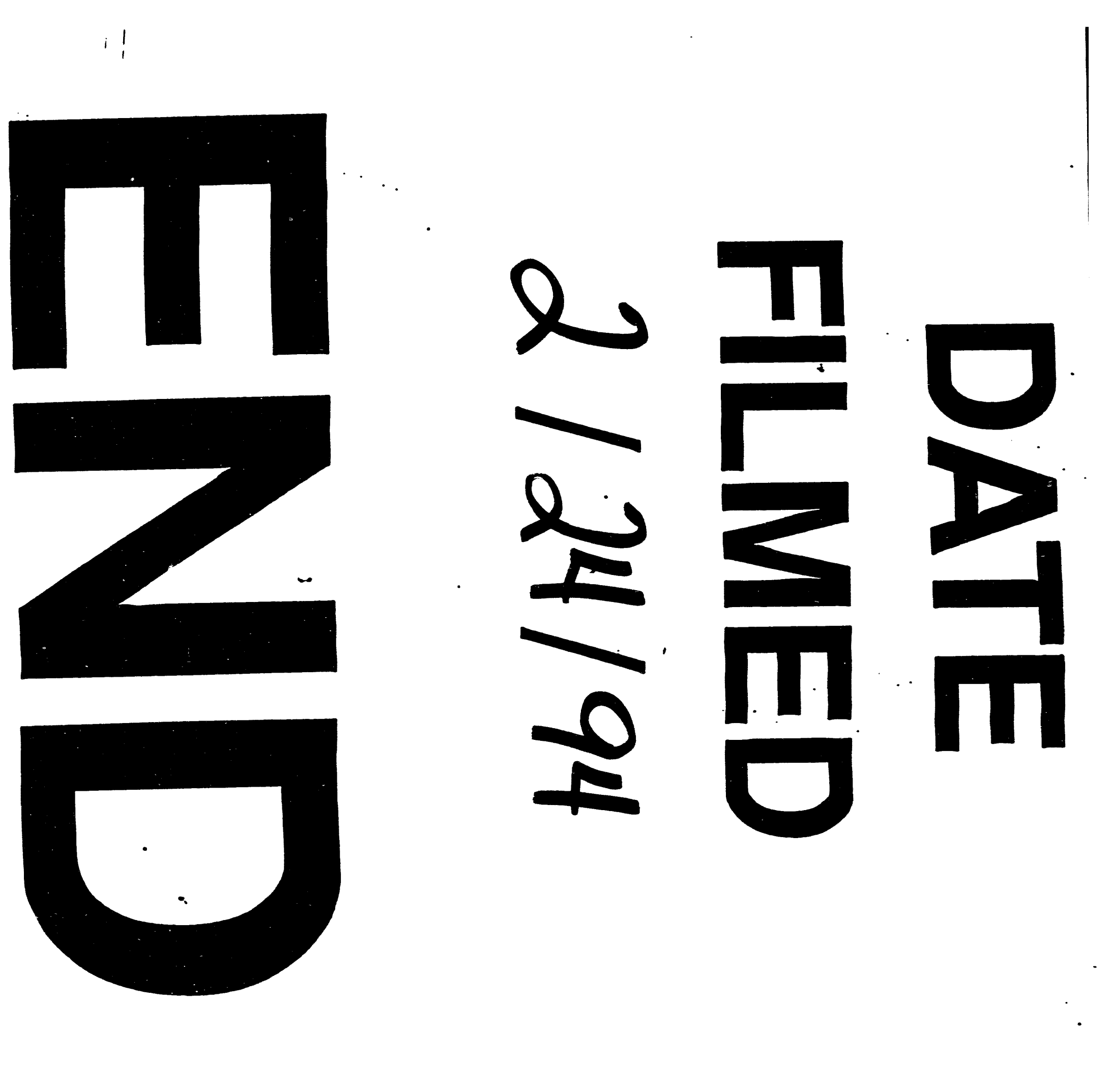


